\title{
Volume terms for charged colloids: A grand-canonical treatment
}

\author{
Bas Zoetekouw and René van Roij \\ Institute for Theoretical Physics, Utrecht University, Leuvenlaan 4, 3584CE Utrecht, The Netherlands
}

(Received 10 October 2005; published 22 February 2006)

\begin{abstract}
We present a study of thermodynamic properties of suspensions of charged colloids on the basis of linear Poisson-Boltzmann theory. We calculate the effective Hamiltonian of the colloids by integrating out the ionic degrees of freedom grand canonically. This procedure not only yields the well-known pairwise screenedCoulomb interaction between the colloids, but also additional volume terms that affect the phase behavior and the thermodynamic properties, such as the osmotic pressure. These calculations are greatly facilitated by the grand-canonical character of our treatment of the ions and allow for relatively fast computations compared to earlier studies in the canonical ensemble. Moreover, the present derivation of the volume terms are relatively simple, make a direct connection with Donnan equilibrium, yield an explicit expression for the effective screening constant, and allow for extensions to include, for instance, nonlinear effects.
\end{abstract}

DOI: 10.1103/PhysRevE.73.021403

PACS number(s): 82.70.Dd, 64.60.Cn, 64.10. + h, 82.39.Wj

\section{INTRODUCTION}

Colloidal suspensions are multicomponent systems that consist of mesoscopic colloidal particles dispersed in a molecular solvent. Often other chemical components are present as well, e.g., ions, polymers, or proteins. Predicting or understanding the properties of such mixtures from a microscopic perspective is generally complicated because the large asymmetry in size and charge between the colloids and the other components, in practice, inhibits a treatment of all the components on an equal footing. The standard way out is to regard the suspension as an effective colloids-only system in which all microscopic degrees of freedom of the "medium" (solvent, ions, polymers, etc.) are suitably averaged out.

For instance, in the case of colloidal hard spheres in a medium with nonadsorbing ideal polymers (radius of gyration $R_{\mathrm{g}}$ ) the so-called depletion effect $[1,2]$ leads to effective attractions between pairs of colloids at surface-surface separations less than $2 R_{\mathrm{g}}$, and in the case of charged colloidal spheres in an electrolyte with Debye length $\kappa^{-1}$ the effective interaction between a colloidal pair at center-to-center separation $r$ is generally written as a repulsive screened-Coulomb potential $\propto \exp (-\kappa r) / r$. The advantage of such a onecomponent viewpoint is that all of the machinery of classical one-component fluids (integral equations, perturbation theory, simulation, etc.) can be employed to study the properties of colloidal suspensions, but only after a reliable averaging over the medium has been performed. Performing this averaging explicitly is generally a tremendous statistical mechanics problem that can only be solved approximately, in most cases [3-5].

One important problem is that the effective colloidal interactions are not necessarily pairwise additive, i.e., triplet or higher-order many-body potentials may appear even if the underlying interactions in and with the medium are strictly pairwise. On physical grounds one generally expects the breakdown of pairwise additivity of the effective interactions if the typical length scale in the medium is of the order of the typical colloidal length scale, e.g., the colloidal radius $a$. For colloid-polymer mixtures, it was indeed shown that equal- sized colloids and polymers $\left(R_{\mathrm{g}}=a\right)$ have bulk and interfacial properties that differ dramatically from pairwise predictions $[6,7]$, and charged colloids in an electrolyte were shown to exhibit non-negligible effective triplet attractions on top of the pairwise repulsions [8] at (extremely) low salt concentrations where $\kappa^{-1} \sim a$.

In this paper we will focus on a description of effective interactions (or the effective Hamiltonian) in bulk suspensions of charged colloids. The classical theory for these systems dates back to the 1940s, when Derjaguin and Landau [9] and Verwey and Overbeek [10] independently calculated that the effective potential between two identical homogeneously charged colloidal spheres (radius $a$, total charge $-Z e$ with $e$ the proton charge) in a bulk medium with dielectric constant $\epsilon$ and Debye length $\kappa^{-1}$ is given by

$$
V_{2}(r)= \begin{cases}\infty & r<2 a \\ \frac{Z^{2} e^{2}}{\epsilon}\left(\frac{\exp (\kappa a)}{1+\kappa a}\right)^{2} \frac{\exp (-\kappa r)}{r} ; & r>2 a .\end{cases}
$$

Here and in the remainder of this paper, we ignore the dispersion forces and recall that $\kappa$ is defined as

$$
\kappa^{2}=8 \pi \lambda_{\mathrm{B}} c_{\mathrm{s}}
$$

in the case of a 1:1 electrolyte with total ion concentration $2 c_{\mathrm{s}}$ far from the colloids, where $\lambda_{\mathrm{B}}=\beta e^{2} / \epsilon$ is the Bjerrum length, $\beta=1 / k_{\mathrm{B}} T$, and $T$ the temperature $[9,10]$.

It is well established by now that many properties of suspensions of $N \gg 1$ charged colloids in a solvent volume $V$ (density $n=N / V$ ) can be understood on the basis of the pairwise effective Hamiltonian

$$
H_{2}(\{\mathbf{R}\})=\sum_{i<j}^{N} V_{2}\left(R_{i j}\right),
$$

where $\mathbf{R}_{i}$ denotes the position of colloid $i=1, \ldots, N$ and $R_{i j}=\left|\mathbf{R}_{i}-\mathbf{R}_{j}\right|$. For instance, the thermodynamic equilibrium properties and the phase behavior follow from the Helmholtz free energy $F_{2}(N, V, T)$, defined as the classical canonical phase-space integral 


$$
\begin{aligned}
\exp \left(-\beta F_{2}\right) & =\frac{1}{N ! \mathcal{V}^{N}} \int_{V} \mathrm{~d} \mathbf{R}^{N} \exp \left(-\beta H_{2}\right) \\
& \equiv \operatorname{tr}_{\mathrm{c}} \exp \left(-\beta H_{2}\right),
\end{aligned}
$$

where $\mathcal{V}$ is an irrelevant constant volume (accounting for the internal partition function of the colloids), which we include for dimensional reasons, and where we introduced the shorthand notation $\operatorname{tr}_{\mathrm{c}}$ for the classical canonical trace over the colloid degrees of freedom. On the basis of Eqs. (1), (3), and (4), one can explain many experimental observations, including the crystallization of (essentially) hard spheres ( $Z=0$ or $\kappa a \gg 1)$ at packing fractions $\eta=4 \pi a^{3} n / 3>0.5$ into an fcc crystal $[11,12]$, the crystallization into bcc crystals for sufficiently soft spheres $[13,14]$, the measured osmotic equation of state $[15,16]$, structure factor $[17,18]$, radial distribution function [19], pair interactions [20,21], and many other colloidal phenomena. It is therefore fair to state that the Derjaguin-Landau-Verwey-Overbeek (DLVO) theory as described by the Eqs. (1), (3), and (4) is one of the cornerstones of colloid science.

It is, however, also fair to add that not all experimental observations are in (qualitative) agreement with DLVO theory. For instance, the experimental observation of "voids" and "Swiss-cheese" structures in otherwise homogeneous suspensions have been interpreted as manifestations of gasliquid coexistence [22,23], and the small lattice spacing of colloidal crystals compared to the one expected on the basis of the known density $n$ was interpreted as evidence for gascrystal coexistence [24]. These possibilities seemed to be confirmed by direct observations of (meta-)stable gas-crystal coexistence [25], and a macroscopic gas-liquid meniscus [26], although these observations were disputed by others $[27,28]$.

Despite the ongoing debates due to a lack of experimental consensus, these experimental results, which were all performed at low ionic strength with $c_{\mathrm{s}}$ in the micromolar regime, triggered a lot of theoretical activities to find the source of cohesive energy that stabilizes the dense liquid or crystal phase in coexistence with a much more dilute gas phase. The dispersion forces would be the first natural candidate to provide the cohesion, but their approximate nanometer range is generally considered to be too small to dominate over the electrostatic repulsions with a range of $\kappa^{-1}$ $\sim 100 \mathrm{~nm}$ at these low salt concentrations.

It was, for instance, found that ion-ion correlations, which are ignored in the derivation of $V_{2}(r)$, can lead to attractive contributions to the pair potential. However, the effect is small and too short ranged for monovalent ions at room temperature in water [13].

Another avenue of research considered the possibility of the breakdown of pairwise additivity due to non-negligible effective triplet and higher-order forces. Within PoissonBoltzmann theory, the triplet potential was calculated and turned out to be attractive, indeed [8], thereby suggesting that many-body interactions could be the source of cohesive energy. Phase diagrams based on repulsive pair interactions (1) and the attractive triplet potential indeed showed coexistence of a dilute gas with very dense crystal phases (as well as crystal-crystal coexistence) $[29,30]$, while experimental evidence for the breakdown of pairwise additivity was obtained by an inverse Ornstein-Zernike analysis of measured colloidal radial distribution functions [31-33], as well as by direct measurement $[34,35]$. Thus, although pairwise additivity seems to be breaking down at low salinity, it is yet questionable whether an approach based on the explicit calculation of triplet and higher-order potentials, if feasible at all, is very efficient, as convergence is probably slow in the regime of strong triplet attractions: there is hardly any justification to ignore the four-body potential when including the triplet potential changes the phase diagram completely compared to the pairwise case. This notion was made explicit by a recent simulation study of the primitive model (charged colloids and explicit microions) that underlies the effective onecomponent system of Ref. [30]: the gas-crystal coexistence that was found with included triplet interactions disappeared again in the simulations of the multicomponent simulation [36].

An alternative representation of nonpairwise interactions is based on density-dependent pair potentials. Roughly speaking, this implies that the explicit coordinate dependence of higher-body potentials is smeared out to reduce to density dependence in the pair interactions. In the case of charged colloids, it seems natural to modify the form of the screening constant such that not only the background (reservoir) salt concentration $2 c_{\mathrm{s}}$ but also the finite concentration $\mathrm{Zn}$ of the counterions and the hard-core exclusion from the colloidal volume is taken into account. For instance, one replaces $\kappa$ by $\widetilde{\kappa}=\sqrt{4 \pi \lambda_{\mathrm{B}}\left(2 c_{\mathrm{S}}+Z n\right)}, \sqrt{4 \pi \lambda_{\mathrm{B}}\left(2 c_{\mathrm{S}}+Z n\right) /(1-\eta)}$ or similar expressions $[13,37-44]$ that reduce in the dilute limit $n \rightarrow 0$ to $\kappa$ as given by Eq. (2). Often $\widetilde{\kappa}(n)>\kappa$, and one could interpret the resulting reduction of the pairwise repulsions due to the more efficient screening at higher density as an effective attractive many-body effect.

Interestingly, however, a careful analysis of the total free energy of the suspension reveals that a density-dependent screening constant affects not only the pair interactions but also one-body contributions, such as the free energy of each colloid with its "own" diffuse cloud of counterions [38-43,45-47]. The thickness of this double layer is typically $\widetilde{\kappa}^{-1}$, and hence, its typical (free) energy is of the order of $-(Z e)^{2} / \epsilon\left(a+\widetilde{\kappa}^{-1}\right)$ (i.e., the Coulomb energy of two charges $\pm Z e$ at separation $\left.a+\widetilde{\kappa}^{-1}\right)$. This term lowers progressively with increasing $n$ and thus provides cohesive energy, whereas it is an irrelevant constant offset of the free energy if a constant $\kappa$ is taken instead of $\widetilde{\kappa}(n)$. It was shown that the density dependence of these so-called volume terms could drive a gas-liquid spinodal instability at low salt concentrations [38,40,46,47] and could hence (qualitatively) explain some of the puzzling experimental observations.

There are several reasons, however, to revisit the theories of, e.g., Refs. [38,40,46,47]. First of all, they are formulated in the canonical ensemble (fixed ion concentrations), which not only obscures its close relationship with the classical Donnan theory for colloidal suspensions $[48,49]$, but also unnecessarily complicates the numerical calculation of phase diagrams as we will argue in Sec. II.

Moreover, and more importantly, the derivation of the explicit expressions for the total free energy was perhaps not 
very transparent in Refs. [46,47], and may have hindered extensions of the theory to include, for instance, charge renormalization. This nonlinear effect was first studied in a cell geometry [50] and, more recently, in a jelliumlike model [51,52]. In both of those models, the nonlinear character of the theory is retained, while its complicated multicentered nature is replaced by a radially symmetric structure. The effective colloidal charge $Z^{*}$ that appears in the prefactor of the DLVO repulsions is then reduced from its bare value $Z$ due to a tightly adsorbed layer of counterions in the vicinity of the colloidal surface. This effect is important when $Z \lambda_{\mathrm{B}} / a$ $\gg 1[50,53-56]$ and therefore casts serious doubt [57] on the predictions of the gas-liquid and gas-crystal transitions in, e.g., Refs. $[38,46,47]$ since large values of $Z$ were needed to have the transitions $[52,58]$. If one now realizes that $Z^{*}$ depends on $n$ and $\widetilde{\kappa}(n)$, as was shown in, e.g., Ref. [53], it is easy to imagine that the volume terms are affected nontrivially by charge renormalization similarly as by the $n$ dependence of the screening parameter. It is therefore important to be able to include this effect into volume-term-type theories and hence to reformulate these theories as transparently as possible.

In order to be able to address all these issues, we revisit here the purely linear screening theory with volume terms. Its nonlinear extension to include charge renormalization will be discussed in a forthcoming paper [59]. The present paper is organized as follows. In Sec. II, we introduce the microscopic Hamiltonian $\mathcal{H}$ of the colloid-ion mixture and give formal expressions for the effective Hamiltonian $H$ for the colloids. In Sec. III, we calculate $H$ by minimizing the mean-field grand potential functional of the ions, whereby explicit expression for the density-dependent screening parameter, the Donnan potential, and the Donnan effect are obtained as intermediate results. In Sec. IV, we consider the thermodynamics of the suspension, in particular, the free energy and the osmotic pressure, with a few interesting canceling contributions. In Sec. V, we calculate a few phase diagrams. We conclude and summarize in Sec. VI.

\section{HAMILTONIAN, DONNAN ENSEMBLE, AND EFFECTIVE HAMILTONIAN}

We consider a suspension of $N$ identical colloidal spheres (radius $a$, positions $\mathbf{R}_{i}$, charge $-Z e$ homogeneously distributed on the surface) in a continuum solvent of volume $V$ characterized by a dielectric constant $\epsilon$ at temperature $T$. The density of the colloids is denoted by $n=N / V$. In addition there are $N_{+}$and $N_{-}$monovalent point like cations $(+)$and anions (-) present, respectively, and charge neutrality dictates that $N_{+}=N_{-}+Z N$. The total interaction Hamiltonian of the system can therefore be written as

$$
\mathcal{H}=\mathcal{H}_{\mathrm{cc}}+\mathcal{H}_{\mathrm{cs}}+\mathcal{H}_{\mathrm{ss}}
$$

where the bare colloid-colloid Hamiltonian $\mathcal{H}_{\mathrm{cc}}$, the colloidsalt Hamiltonian $\mathcal{H}_{\mathrm{cs}}$, and the salt-salt Hamiltonian $\mathcal{H}_{\mathrm{ss}}$ are pairwise sums of hard-core and (unscreened) Coulomb potentials. We write $\mathcal{H}_{\mathrm{cc}}=\sum_{i<j}^{N} V_{\mathrm{cc}}\left(R_{i j}\right)$ with

$$
\beta V_{\mathrm{cc}}(r)= \begin{cases}\infty & r<2 a ; \\ \frac{Z^{2} \lambda_{\mathrm{B}}}{r} & r>2 a,\end{cases}
$$

and $\mathcal{H}_{\mathrm{cs}}=\mathcal{H}_{\mathrm{c}+}+\mathcal{H}_{\mathrm{c}-}$ with $\mathcal{H}_{\mathrm{c} \pm}=\sum_{i=1}^{N} \Sigma_{j=1}^{N_{ \pm}} V_{\mathrm{c} \pm}\left(\left|\mathbf{R}_{i}-\mathbf{r}_{j}^{ \pm}\right|\right)$, where

$$
\beta V_{c \pm}(r)= \begin{cases}\infty & r<a ; \\ \mp \frac{Z \lambda_{\mathrm{B}}}{r} & r>a,\end{cases}
$$

and $\mathbf{r}_{j}^{ \pm}$is the position of the $j$ th positive (negative) microion. The expression for $\mathcal{H}_{\mathrm{ss}}$ is similar, but without the hard-core terms because of the pointlike nature of the ions.

In principle, the thermodynamic properties of this system could be calculated from the Helmholtz free energy of the system $\mathcal{F}\left(N, N_{-}, V, T\right)$, which is defined by $\exp (-\beta \mathcal{F})$ $=\operatorname{tr}_{\mathrm{c}} \mathrm{tr}_{+} \mathrm{tr}_{-} \exp (-\beta \mathcal{H})$. The canonical traces are defined as in Eq. (4). Note that one can ignore the explicit $N_{+}$dependence of $\mathcal{F}$ because of the charge neutrality condition.

Within linearized Poisson-Boltzmann theory and exploiting the Gibbs-Bogolyubov inequality, $\mathcal{F}$ was explicitly calculated in Refs. [46,47]. The phase diagram was then constructed from $\mathcal{F}$ by imposing the usual conditions of mechanical and diffusive equilibrium, viz.

$$
\left\{\begin{array}{l}
P\left(n^{(1)}, n_{-}^{(1)}\right)=P\left(n^{(2)}, n_{-}^{(2)}\right) \\
\mu\left(n^{(1)}, n_{-}^{(1)}\right)=\mu\left(n^{(2)}, n_{-}^{(2)}\right) \\
\mu_{-}\left(n^{(1)}, n_{-}^{(1)}\right)=\mu_{-}\left(n^{(2)}, n_{-}^{(2)}\right),
\end{array}\right.
$$

where $n^{(i)}$ and $n_{-}^{(i)}$ denote the colloid density and the anion density in phase $\bar{i}$, respectively, and where we introduced the pressure $P=-(\partial \mathcal{F} / \partial V)$, the colloidal chemical potential $\mu$ $=(\partial \mathcal{F} / \partial N)$, and the anion chemical potential $\mu_{-}=\left(\partial \mathcal{F} / \partial N_{-}\right)$. The system (8) of three equations for the four unknown densities yielded the phase diagram in the $n-n_{-}$plane, for given parameters $Z, a$, and $\lambda_{\mathrm{B}}$.

These canonical ensemble calculations were, however, numerically rather demanding, since many numerically expensive evaluations of $P\left(n, n_{-}\right), \mu\left(n, n_{-}\right)$, and $\mu_{-}\left(n, n_{-}\right)$are needed in the root-finding procedure of solving Eqs. (8). For that reason the phase diagram of only a few combinations of parameters $Z, \lambda_{\mathrm{B}}$, and $a$ has been studied in some detail. Moreover, the derivation of the explicit expressions for $\mathcal{F}$ was, perhaps, not very transparent and may have hindered extensions of the theory to include, for instance, nonlinear effects such as charge renormalization. Additionally, the (strong) connection with the standard description of colloidal suspensions in terms of a Donnan equilibrium was not made in Refs. [46,47].

It turns out, as we will show in this paper, that at least some of these shortcomings and drawbacks of working in the canonical ensemble can be lifted by treating the anions and cations grand canonically. For this we assume the suspension to be in diffusive contact with a dilute reservoir of monovalent anions and cations at chemical potential $\mu_{ \pm}$ $=k_{\mathrm{B}} T \ln \left(c_{\mathrm{s}} \Lambda_{ \pm}^{3}\right)$, where $2 c_{\mathrm{s}}$ is the total ion density in the (charge neutral) reservoir, and where $\Lambda_{ \pm}$is the thermal wavelength of the cations $(+)$ and anions $(-)$, respectively. The colloidal particles cannot enter the ion reservoir (e.g., be- 
cause of a semipermeable membrane in an actual experimental setting) and remain treated canonically (fixed $N$ and $V$ ) as before. The thermodynamic potential of this ensemble, which we will call the "Donnan ensemble" from now on, is denoted $F=\mathcal{F}-\mu_{+} N_{+}-\mu_{-} N_{-}$, and is a function of the variables $N, V, T$, and $\mu_{ \pm}$. It is related to the microscopic Hamiltonian by the "Donnan partition function"

$$
\exp (-\beta F)=\operatorname{tr}_{\mathrm{c}} \operatorname{Tr}_{+} \operatorname{Tr}_{-} \exp (-\beta \mathcal{H}),
$$

where $\mathcal{H}$ was defined in Eq. (5) and the grand-canonical traces are defined as

$$
\operatorname{Tr}_{ \pm}=\sum_{i=1}^{N_{ \pm}} \exp \left(\beta \mu_{ \pm} N_{ \pm}\right) \operatorname{tr}_{ \pm}=\sum_{i=1}^{N_{ \pm}} \frac{c_{\mathrm{s}}^{N_{ \pm}}}{N_{ \pm} !} \int \mathrm{d} \mathbf{r}_{ \pm}^{N_{ \pm}}
$$

Here we have used that $\exp \left(\beta \mu_{ \pm}\right) / \Lambda_{ \pm}^{3}=c_{\mathrm{s}}$ (where the factor $1 / \Lambda_{ \pm}^{3}$ follows from the classical momentum integration), and we denoted the microion coordinates by $\mathbf{r}_{ \pm}^{N_{ \pm}}$. For convenience we will drop the explicit $T$ dependence from now on and replace the dependence on $\mu_{ \pm}$by the reservoir concentration $c_{\mathrm{s}}$.

Because of the extensive character of $F$, we can write $F\left(N, V, c_{\mathrm{s}}\right)=V f\left(n, c_{\mathrm{s}}\right)$. The thermodynamic properties follow now as $\mu=(\partial F / \partial N)=(\partial f / \partial n)$ and $P=-(\partial F / \partial V)=n \mu-f$, where the derivatives are to be taken at fixed $c_{\mathrm{s}}$ and $T$. This implies that the phase-coexistence conditions simplify to the two conditions

$$
\left\{\begin{array}{l}
P\left(n^{(1)}, c_{\mathrm{s}}\right)=P\left(n^{(2)}, c_{\mathrm{s}}\right) ; \\
\mu\left(n^{(1)}, c_{\mathrm{s}}\right)=\mu\left(n^{(2)}, c_{\mathrm{s}}\right),
\end{array}\right.
$$

for the two unknown colloid densities $n^{(i)}$, at fixed $c_{\mathrm{s}}$, i.e., we have prearranged equal chemical potential of the ions due to our choice to work in the Donnan ensemble. This is a considerable reduction of the numerical effort compared to Eqs. (8). Note that the mechanical equilibrium condition is equivalent to equal osmotic pressure $\Pi$ in the two coexisting phases, where $\Pi\left(n, c_{\mathrm{s}}\right)=P\left(n, c_{\mathrm{s}}\right)-P\left(0, c_{\mathrm{s}}\right)$ is the suspension's excess pressure over the reservoir pressure $P\left(0, c_{\mathrm{s}}\right)=2 c_{\mathrm{s}} k_{\mathrm{B}} T$ (recall that we treated the reservoir as an ideal gas here). This simple relation allows for a rather direct contact with experimental measurements of the osmotic pressure, as we will also show below.

Even though our main objective is to calculate $F\left(N, V, c_{\mathrm{s}}\right)$ as defined in Eq. (9), we will first focus on an important and convenient intermediate quantity: the effective Hamiltonian $H$, which depends on the colloid configuration $\{\mathbf{R}\}$ and parametrically on the reservoir salt concentration $c_{\mathrm{s}}$. It is defined as

$$
\begin{aligned}
\exp (-\beta H) & =\operatorname{Tr}_{+} \operatorname{Tr}_{-} \exp (-\beta \mathcal{H}) \\
& =\exp \left(-\beta \mathcal{H}_{\mathrm{cc}}\right) \operatorname{Tr}_{+} \operatorname{Tr}_{-} \exp \left(-\beta \mathcal{H}_{\mathrm{cs}}-\beta \mathcal{H}_{\mathrm{ss}}\right) \\
& \equiv \exp \left(-\beta \mathcal{H}_{\mathrm{cc}}\right) \exp (-\beta \Omega)
\end{aligned}
$$

where, in the last step, we defined the grand partition function $\exp (-\beta \Omega)$ of the inhomogeneous system of interacting cations and anions (through $\mathcal{H}_{\mathrm{ss}}$ ) in the external potential of the colloids (through $\mathcal{H}_{\mathrm{cs}}$ ). The corresponding grand potential of this system is $\Omega$, which is the quantity that we need to calculate in order to find the effective Hamiltonian given from Eq. (12) as

$$
H=\mathcal{H}_{c c}+\Omega .
$$

Once $H$ is known, we can use standard one-component techniques to obtain approximate expressions for $F$, since $\exp (-\beta F)=\operatorname{tr}_{\mathrm{c}} \exp (-\beta H)$ is precisely as if $F$ were the Helmholtz free energy of a one-component system with Hamiltonian $H$.

\section{GRAND POTENTIAL $\Omega$}

\section{A. Density functional}

We will not explicitly calculate $\Omega$ from the grand partition function of Eq. (12). Instead, we exploit the framework of classical density functional theory (DFT), which treats an inhomogeneous fluid in an external field at the level of the one-body distribution functions (the density profiles) [60-62]: the equilibrium density profiles minimize the (variational) grand potential functional, and this minimum is the grand potential. Here we denote the density profile of the cations by $\rho_{+}(\mathbf{r})$, that of the anions by $\rho_{-}(\mathbf{r})$, and the grandpotential functional by $\Omega\left[\rho_{+}, \rho_{-}\right]$. For notational convenience, we do not introduce a separate notation for the variational and equilibrium profiles, and neither for the grandpotential functional and its minimum (equilibrium) value.

The cations and anions experience external potentials $U_{+}(\mathbf{r})$ and $U_{-}(\mathbf{r})$, respectively, due to the Coulomb and excluded volume interactions with a fixed configuration $\{\mathbf{R}\}$ of colloidal particles. These potentials are explicitly given by

$$
U_{ \pm}(\mathbf{r})=\sum_{i=1}^{N} V_{c \pm}\left(\left|\mathbf{R}_{i}-\mathbf{r}\right|\right),
$$

where the colloid-ion pair potentials $V_{\mathrm{c} \pm}(r)$ were defined in Eq. (7). We can now write the grand potential functional within a simple mean-field approximation as

$$
\begin{aligned}
\Omega\left[\rho_{+}, \rho_{-}\right]= & \Omega_{\mathrm{id}}\left[\rho_{+}\right]+\Omega_{\mathrm{id}}\left[\rho_{-}\right]+\frac{e^{2}}{2 \epsilon} \int \mathrm{d} \mathbf{r} \mathrm{d} \mathbf{r}^{\prime} \frac{\rho(\mathbf{r}) \rho\left(\mathbf{r}^{\prime}\right)}{\left|\mathbf{r}-\mathbf{r}^{\prime}\right|} \\
& +\int \mathrm{d} \mathbf{r}\left[\rho_{+}(\mathbf{r}) U_{+}(\mathbf{r})+\rho_{-}(\mathbf{r}) U_{-}(\mathbf{r})\right],
\end{aligned}
$$

where we defined the ion charge density $\rho(\mathbf{r})=\rho_{+}(\mathbf{r})-\rho_{-}(\mathbf{r})$, and where the ideal-gas grand potential functional can be written as

$$
\begin{aligned}
\Omega_{\mathrm{id}}\left[\rho_{ \pm}\right] & =\int \mathrm{d} \mathbf{r} \rho_{ \pm}(\mathbf{r})\left\{-\mu_{ \pm}+k_{\mathrm{B}} T\left[\ln \rho_{ \pm}(\mathbf{r}) \Lambda_{ \pm}^{3}-1\right]\right\} \\
& =k_{\mathrm{B}} T \int \mathrm{d} \mathbf{r} \rho_{ \pm}(\mathbf{r})\left(\ln \frac{\rho_{ \pm}(\mathbf{r})}{c_{\mathrm{s}}}-1\right)
\end{aligned}
$$

Here we have substituted the identity $\mu_{ \pm}=k_{\mathrm{B}} T \ln c_{\mathrm{s}} \Lambda_{ \pm}^{3}$.

The Euler-Lagrange equations $\delta \Omega / \delta \rho_{ \pm}(\mathbf{r})=0$ that correspond with Eq. (15), can be cast, for $\mathbf{r}$ outside a colloidal hard core, into the form $\rho_{ \pm}(\mathbf{r})=c_{\mathrm{s}} \exp [\mp \phi(\mathbf{r})]$. The dimensionless potential $\phi(\mathbf{r})$ must then satisfy the nonlinear multicentered Poisson-Boltzmann equation [63] 


$$
\nabla^{2} \phi(\mathbf{r})=\kappa^{2} \sinh \phi(\mathbf{r})-\frac{Z \lambda_{\mathrm{B}}}{a^{2}} \sum_{i=1}^{N} \delta\left(\left|\mathbf{r}-\mathbf{R}_{i}\right|-a\right),
$$

where $\delta(\mathbf{r})$ is the Dirac $\delta$. Unfortunately, no analytical solution to Eq. (17) is known for the multicentered geometry of interest here. Even solving Eq. (17) numerically is far from trivial and requires a serious computational effort [64-69].

For this reason, we will first make further approximations to the functional and then perform its minimization afterward. The main approximation involves the expansion, up to quadratic order, of the ideal-gas grand potential terms about the, as of yet unknown, ion densities $\bar{\rho}_{ \pm}$, such that $\rho_{ \pm}(\mathbf{r})$ $-\bar{\rho}_{ \pm}$are considered to be the "small" expansion parameters. This expansion yields $\Omega_{\mathrm{id}}\left[\rho_{ \pm}\right] \approx \Omega_{\mathrm{id}}^{\prime}\left[\rho_{ \pm}\right]$with

$$
\begin{aligned}
\beta \Omega_{\mathrm{id}}^{\prime}\left[\rho_{ \pm}\right]= & \bar{\rho}_{ \pm}\left(\ln \frac{\bar{\rho}_{ \pm}}{c_{\mathrm{S}}}-1\right) V+\ln \frac{\bar{\rho}_{ \pm}}{c_{\mathrm{S}}} \int \mathrm{d} \mathbf{r}\left[\rho_{ \pm}(\mathbf{r})-\bar{\rho}_{ \pm}\right] \\
& +\frac{1}{2 \bar{\rho}_{ \pm}} \int \mathrm{d} \mathbf{r}\left[\rho_{ \pm}(\mathbf{r})-\bar{\rho}_{ \pm}\right]^{2} .
\end{aligned}
$$

In principle, this expansion holds for arbitrary $\bar{\rho}_{ \pm}$, but later on we will choose $\bar{\rho}_{ \pm}$to be equal to the average ion concentrations in the system, such that $\int \operatorname{dr}\left[\rho_{ \pm}(\mathbf{r})-\bar{\rho}_{ \pm}\right]=0$, i.e., $V \bar{\rho}_{ \pm}=N_{ \pm}$is the number of ions in the suspension. As will be shown below, this linearization corresponds to a linearization of Eq. (17) about $\phi(\mathbf{r})=\bar{\phi}$ with $\bar{\phi}$ the Donnan potential. This is in line with Ref. [70].

It turns out to be convenient, and necessary, to rewrite the external potentials $U_{ \pm}(\mathbf{r})$ for the ions such that $U_{ \pm}(\mathbf{r})$ $= \pm V(\mathbf{r})+W(\mathbf{r})$, where we defined the electrostatic potential (due to the colloids) $V(\mathbf{r})=\Sigma_{i} v\left(\left|\mathbf{r}-\mathbf{R}_{i}\right|\right)$ and the hard-core potential $W(\mathbf{r})=\sum_{i} w\left(\left|\mathbf{r}-\mathbf{R}_{i}\right|\right)$, with

$$
\beta v(r)= \begin{cases}\beta v_{0} & r<a ; \\ -Z \lambda_{\mathrm{B}} / r & r>a,\end{cases}
$$

and

$$
\beta w(r)= \begin{cases}\beta w_{0} & r<a ; \\ 0 & r>a .\end{cases}
$$

Although we are actually interested in the hard-core limit $\beta v_{0} \rightarrow \infty$ and $\beta w_{0} \rightarrow \infty$ here, we introduce the (finite) hardcore parameters $v_{0}$ and $w_{0}$ here for later convenience. They are necessary and sufficient to ensure, within the linearized theory, a vanishing ion density in the colloidal hard cores.

Collecting the results, we can write the approximate grand potential functional as

$$
\begin{aligned}
\Omega\left[\rho_{+}, \rho_{-}\right]= & \Omega_{\mathrm{id}}^{\prime}\left[\rho_{+}\right]+\Omega_{\mathrm{id}}^{\prime}\left[\rho_{-}\right]+\frac{e^{2}}{2 \epsilon} \int \mathrm{d} \mathbf{r} \mathrm{d} \mathbf{r}^{\prime} \frac{\rho(\mathbf{r}) \rho\left(\mathbf{r}^{\prime}\right)}{\left|\mathbf{r}-\mathbf{r}^{\prime}\right|} \\
& +\int \mathrm{d} \mathbf{r}\left\{\rho(\mathbf{r}) V(\mathbf{r})+\left[\rho_{+}(\mathbf{r})+\rho_{-}(\mathbf{r})\right] W(\mathbf{r})\right\},
\end{aligned}
$$

which is minimized by those (equilibrium) profiles that satisfy the Euler-Lagrange equations

$$
\ln \frac{\bar{\rho}_{ \pm}}{c_{\mathrm{S}}}+\frac{\rho_{ \pm}(\mathbf{r})-\bar{\rho}_{ \pm}}{\bar{\rho}_{ \pm}} \pm \phi(\mathbf{r})+\beta W(\mathbf{r})=0 .
$$

Here we introduced the (dimensionless) electrostatic potential $\phi(\mathbf{r})$, given by

$$
\phi(\mathbf{r})=\lambda_{\mathrm{B}} \int \mathrm{d} \mathbf{r}^{\prime} \frac{\rho\left(\mathbf{r}^{\prime}\right)}{\left|\mathbf{r}-\mathbf{r}^{\prime}\right|}+\beta V(\mathbf{r}) .
$$

\section{B. Equilibrium profiles and Donnan equilibrium}

We leave the hard-core parameters $v_{0}$ and $w_{0}$ undetermined for now and start the analysis of the Euler-Lagrange equations by integrating Eq. (21) over the volume. At the same time, we impose that $\int \mathrm{d} \mathbf{r}\left[\rho_{ \pm}(\mathbf{r})-\bar{\rho}_{ \pm}\right]=0$, i.e., we choose $\bar{\rho}_{ \pm}$such that it is the actual average ion density in the suspension. After rearrangement, we find that

$$
\bar{\rho}_{ \pm}=c_{\mathrm{S}} \exp \left[\overline{\bar{\phi}}-\eta \beta w_{0}\right],
$$

where $\bar{\phi}=\int \mathrm{d} \mathbf{r} \phi(\mathbf{r}) / V$ is the spatially averaged electric potential, i.e., the Donnan potential. Since global charge neutrality imposes that $\bar{\rho}_{+}-\bar{\rho}_{-}=Z n$, we can conclude from Eq. (23) that the Donnan potential satisfies

$$
\sinh \bar{\phi}=-\frac{Z n}{2 c_{\mathrm{s}}} \exp \left[\eta \beta w_{0}\right],
$$

which reduces to the usual Donnan expressions in the pointcolloid limit $\eta \rightarrow 0$ [48,71]. Combining Eq. (24) with (23) yields

$$
\bar{\rho}_{ \pm}=\frac{1}{2}\left(\sqrt{(Z n)^{2}+\left(2 c_{\mathrm{s}}\right)^{2} \exp \left(-2 \eta \beta w_{0}\right)} \pm Z n\right),
$$

which explicitly relates the salt concentration in the suspension to the colloid density and the salt reservoir concentration, provided the parameter $w_{0}$ is known.

Using these relations for $\bar{\phi}$ and $\bar{\rho}_{ \pm}$, we consider a specific linear combination of the Euler-Lagrange equations and rewrite Eqs. (21) as

$$
\begin{gathered}
\frac{\rho_{+}(\mathbf{r})}{\bar{\rho}_{+}}+\frac{\rho_{-}(\mathbf{r})}{\bar{\rho}_{-}}-2=-2\left[\beta W(\mathbf{r})-\eta \beta w_{0}\right] ; \\
\rho(\mathbf{r})-\bar{\rho}=-\left(\bar{\rho}_{+}+\bar{\rho}_{-}\right)[\phi(\mathbf{r})-\bar{\phi}]-\bar{\rho}\left[\beta W(\mathbf{r})-\eta \beta w_{0}\right],
\end{gathered}
$$

where we defined the shorthand notation $\bar{\rho}=\bar{\rho}_{+}-\bar{\rho}_{-}=Z n$ for the overall ionic charge density. This particular linear combination was chosen because (i) the charge density is the physical quantity of interest here and (ii) the electric potential is decoupled from the "charge-neutral" density.

It is straightforward to solve the "hard-core" linear combination, Eq. (26a). Imposing that $\rho_{+}(\mathbf{r}) / \bar{\rho}_{+}+\rho_{-}(\mathbf{r}) / \bar{\rho}_{-} \equiv 0$ within the hardcore of any of the colloids [i.e., wherever $\left.W(\mathbf{r})=w_{0}\right]$ yields a value for the hard-core parameter,

$$
\beta w_{0}=\frac{1}{1-\eta},
$$

whereas outside any of the colloidal hard core positions we have 


$$
\frac{\rho_{+}(\mathbf{r})}{\bar{\rho}_{+}}+\frac{\rho_{-}(\mathbf{r})}{\bar{\rho}_{-}}=\frac{2}{1-\eta} .
$$

The solution of the "charge" linear combination, Eq. (26b), is most straightforwardly found by Fourier transformation. For an arbitrary function $f(\mathbf{r})$, we define and denote the Fourier transform as $f_{\mathbf{k}}=\int \operatorname{dr} f(\mathbf{r}) \exp (i \mathbf{k} \cdot \mathbf{r})$. One easily checks from Eq. (26b) that

$$
\begin{aligned}
\rho_{\mathbf{k}}= & (2 \pi)^{3}\left\{\bar{\rho}+\left(\bar{\rho}_{+}+\bar{\rho}_{-}\right) \bar{\phi}+\bar{\rho} \eta \beta w_{0}\right\} \delta(\mathbf{k}) \\
& -\left(\bar{\rho}_{+}+\bar{\rho}_{-}\right) \phi_{\mathbf{k}}-\bar{\rho} W_{\mathbf{k}},
\end{aligned}
$$

where we have from Eq. (19b) that

$$
W_{\mathbf{k}}=\frac{4 \pi a w_{0}}{k^{2}}\left(\frac{\sin (k a)}{k a}-\cos (k a)\right) \sum_{j=1}^{N} e^{i \mathbf{k} \cdot \mathbf{R}_{j}},
$$

and from Eqs. (22) and (19a) that

$$
\begin{aligned}
\phi_{\mathbf{k}}= & 4 \pi \lambda_{\mathrm{B}} \frac{\rho_{\mathbf{k}}}{k^{2}}-\frac{4 \pi a}{k^{2}} \sum_{j=1}^{N} \exp \left(i \mathbf{k} \cdot \mathbf{R}_{j}\right) \\
& \times\left\{\left(\beta v_{0}+Z \frac{\lambda_{\mathrm{B}}}{a}\right) \cos k a-\beta v_{0} \frac{\sin k a}{k a}\right\} .
\end{aligned}
$$

Equations (29) and (31) are two linear equations in the unknowns $\phi_{\mathbf{k}}$ and $\rho_{\mathbf{k}}$, which can be solved straightforwardly. Fixing the remaining hard-core parameter $v_{0}$ to

$$
\beta v_{0}=-Z \frac{\bar{\kappa} \lambda_{\mathrm{B}}}{1+\bar{\kappa} a}+\beta w_{0} \frac{\bar{\rho}_{+}-\bar{\rho}_{-}}{\bar{\rho}_{+}+\bar{\rho}_{-}},
$$

we find that the charge density is given by

$$
\begin{aligned}
\rho_{\mathbf{k}}= & (2 \pi)^{3}\left\{\frac{\bar{\rho}}{1-\eta}+\left(\bar{\rho}_{+}+\bar{\rho}_{-}\right) \bar{\phi}\right\} \frac{k^{2}}{k^{2}+\bar{\kappa}^{2}} \delta(\mathbf{k}) \\
& +\frac{Z}{1+\bar{\kappa} a} \frac{\cos k a+\frac{\bar{\kappa}}{k} \sin k a}{1+k^{2} / \bar{\kappa}^{2}} \sum_{j} e^{i \mathbf{k} \cdot \mathbf{R}_{j},}
\end{aligned}
$$

where the effective Debye screening parameter is defined as

$$
\begin{aligned}
\bar{\kappa} & \equiv \sqrt{4 \pi \lambda_{\mathrm{B}}\left(\bar{\rho}_{+}+\bar{\rho}_{-}\right)} \\
& =\sqrt{4 \pi \lambda_{B}} \sqrt[4]{(Z n)^{2}+\left(2 c_{\mathrm{s}} \exp [-\eta /(1-\eta)]\right)^{2}} .
\end{aligned}
$$

Here we used Eqs. (24), (25), and (27) in rewriting the first into the second line. Note that the factor $\exp [-\eta /(1-\eta)]$ that appears in Eq. (34) can be accurately represented by $(1-\eta)$, with a relative deviation less than 0.01 for $\eta<0.1$ and less than 0.1 for $\eta<0.35$.

The first term in expression (33) is of the form $\propto k^{2} \delta(\mathbf{k})$ and does not contribute to the charge density (35). However, we will see below that this term does, in fact, contribute to the grand potential, as this involves the Coulomb energy $\propto \int \mathrm{d} \mathbf{k} \rho_{\mathbf{k}} / k^{2}$.

The real space representation of the charge density is a multicentered sum $\rho(\mathbf{r})=\Sigma_{i} \rho_{1}\left(\left|\mathbf{r}-\mathbf{R}_{i}\right|\right)$, where the oneparticle density profiles (the "orbitals") have the usual DLVO form $[9,10]$

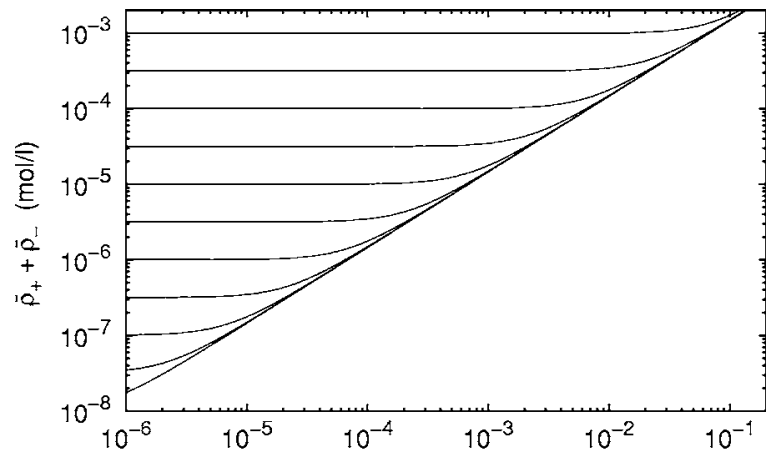

(a)

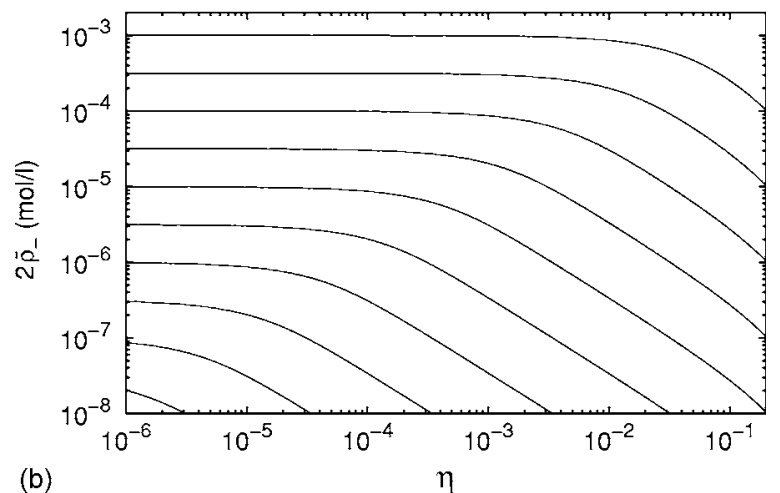

FIG. 1. Total ion concentration $\bar{\rho}_{+}+\bar{\rho}_{-}$(a) and concentration of added salt $\bar{\rho}_{-}$(b) as a function of the colloid packing fraction for different reservoir concentrations, using the expressions of Eqs. (25) and (27). The colloidal charge and radius are $Z=50$ and $a$ $=21.9 \mathrm{~nm}$, respectively, and the solvent is ethanol at room temperature such that $\lambda_{B}=2.37 \mathrm{~nm}$. This matches the parameters from the experiments by Raşa et al. [72].

$$
\rho_{1}(r)= \begin{cases}0 & r<a ; \\ \frac{Z \bar{\kappa}^{2}}{4 \pi} \frac{\exp (\bar{\kappa} a)}{1+\bar{\kappa} a} \frac{\exp (-\bar{\kappa} r)}{r} & r>a .\end{cases}
$$

We note that the vanishing of $\rho_{1}(r)$ inside the colloidal hard core is a direct consequence of our particular choice for $v_{0}$ given by Eq. (32); other choices for $v_{0}$ would have yielded a finite ion charge density inside the hard core. Note also that the multicentered charge density $\rho(\mathbf{r})$ is not vanishing within the hard cores, since the exponential tail of the orbital centered around colloid $i$ penetrates the hard core of all the other colloids $j \neq i$.

By inserting Eq. (27) into (25), explicit expressions for the average concentrations $\bar{\rho}_{ \pm}$of ions in the suspension are obtained as a function of the colloid density $n$, colloid charge $Z$, and the reservoir concentration $2 c_{\mathrm{s}}$ - this was already used to obtain Eq. (34). These expressions reduce, in the limit of pointlike colloids (for which $\eta=0$ ) to the standard expressions for the Donnan effect $[48,49]$.

This effect is illustrated in Fig. 1, where we plot the total ion concentration $\bar{\rho}_{+}+\bar{\rho}_{-}$in Fig. 1(a), and the concentration of added salt $2 \bar{\rho}_{-}=\bar{\rho}_{+}+\bar{\rho}_{-}-Z n$ in Fig. 1(b), on the basis of our expressions for $\bar{\rho}_{ \pm}$. The parameters are close to those of the experiments by Raşa et al. [72] and Raşa and Philipse 
[73]: $Z=50, \lambda_{\mathrm{B}}=2.37 \mathrm{~nm}$, and $a=21.9 \mathrm{~nm}$. The reservoir salt concentration equals the $\eta=0$ limit of each of the curves, and the crossover from the low- $\eta$ plateau to the high- $\eta$ linear part corresponds to the crossover from added-salt dominance to counterion dominance. Note the expulsion of added salt back into the reservoir at high $\eta$ in Fig. 1(b). An important aspect of these intermediate results is that the screening parameter $\bar{\kappa}$ increases with $n$ essentially $\propto \sqrt{Z n}$ in the counterion-dominated regime (which may occur at packing fractions as low as $\eta \simeq 10^{-4}$ if $c_{\mathrm{s}} \simeq 3 \mu \mathrm{M}$ ).

As we have now solved the Euler-Lagrange equations (21) for the two linear combinations $\rho_{+}(\mathbf{r}) / \bar{\rho}_{+}+\rho_{-}(\mathbf{r}) / \bar{\rho}_{-}$and $\rho_{+}(\mathbf{r})-\rho_{-}(\mathbf{r})$, it is straightforward to disentangle the equilibrium profiles and obtain the profiles $\rho_{ \pm}(\mathbf{r})$ of the two ionic species separately.

It is important to realize, however, that these results depend on the particular choice that we have made for the hard-core potentials in Eqs. (19a) and (19b). Different choices for these hard-core potentials lead to other, nonequivalent minima of the grand potential. For instance, instead of $U_{ \pm}(\mathbf{r})= \pm V(\mathbf{r})+W(\mathbf{r})$, we could have considered the choice $U_{ \pm}(\mathbf{r})= \pm V(\mathbf{r})+2 \bar{\rho}_{\overline{+}} W(\mathbf{r}) /\left(\bar{\rho}_{+}+\bar{\rho}_{-}\right)$, which, with $\beta v_{0}$ $=-Z \bar{\kappa} \lambda_{\mathrm{B}} /(1+\bar{\kappa} a)$ and $\beta w_{0}=1 /(1-\eta)$ would lead to a vanishing $\rho_{1}(r)$ and $\rho_{+}(\mathbf{r}) / \bar{\rho}_{+}+\rho_{-}(\mathbf{r}) / \bar{\rho}_{-}$inside the hard cores. This choice was actually made in Refs. $[46,47]$ and leads to similar, but not identical results (see Appendix B).

\section{Minimum of the functional}

In Appendix A, we derive the equilibrium grand potential $\Omega$ by insertion of our solution of the Euler-Lagrange equations into the functional. The effective interaction Hamiltonian $H=\mathcal{H}_{\mathrm{cc}}+\Omega$ then takes the form

$$
H\left(\{\mathbf{R}\}, N, V, c_{\mathrm{s}}\right)=\Phi\left(V, n, c_{\mathrm{s}}\right)+\sum_{i<j}^{N} V\left(R_{i j} ; n, c_{\mathrm{s}}\right) .
$$

The first term $\Phi$, is independent of the colloidal coordinates $\mathbf{R}_{i}$, and is called the "volume term" as it is a densitydependent, extensive thermodynamic quantity that scales with the volume of the system. The second term of Eq. (36) is a pairwise sum that does depend on the colloidal coordinates (and on the density $n$ ). For later convenience we decompose the volume term as $\Phi=\Phi_{\mathrm{D}}+\Phi_{0}$, with the so-called Donnan term defined by

$$
\frac{\beta \Phi_{\mathrm{D}}}{V}=\sum_{ \pm} \bar{\rho}_{ \pm}\left(\ln \frac{\bar{\rho}_{ \pm}}{c_{\mathrm{s}}}-1\right),
$$

and the other term by

$$
\frac{\beta \Phi_{0}}{V}=-\frac{1}{2} \frac{(Z n)^{2}}{\bar{\rho}_{+}+\bar{\rho}_{-}}+\frac{\eta}{1-\eta} \frac{2 \bar{\rho}_{+} \bar{\rho}_{-}}{\bar{\rho}_{+}+\bar{\rho}_{-}}-\frac{n}{2} \frac{Z^{2} \bar{\kappa} \lambda_{\mathrm{B}}}{1+\bar{\kappa} a} .
$$

In Sec. IV B below, we will see that $\Phi_{\mathrm{D}}$, which takes the form of ideal-gas contributions, accounts for the Donnan equation of state (except for the colloidal ideal-gas contribution), hence, the nomenclature. The term $\Phi_{0}$ appears as an electrostatic (and hard-core) free-energy contribution. This separation is slightly misleading, however, since the two

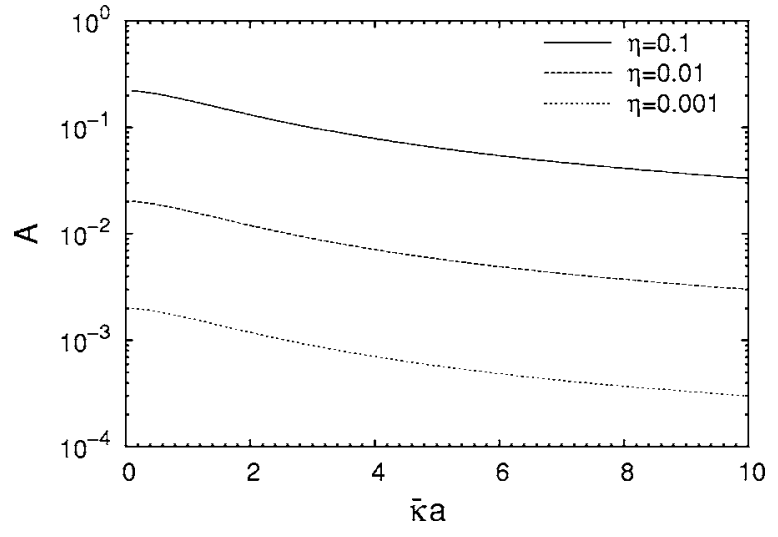

FIG. 2. The factor $A$ of Eq. (40) as a function of $\bar{\kappa} a$ and $\eta$. For all parameters, $A \ll 1$, so it can safely be neglected in Eq. (39).

terms both depend on $n$ and $Z$ through the expressions (25) and (34), which stem from the Donnan potential (24) and, hence, from the balance between electrostatics and entropy.

The effective pair potential between the colloids, $V\left(R_{i j}\right)$, which appears in the second term of Eq. (36), is given by

$$
\beta V(r)= \begin{cases}\infty & r<2 a ; \\ (1+A) Z_{>}^{2} \lambda_{\mathrm{B}} \frac{\exp (-\bar{\kappa} r)}{r} & r>2 a,\end{cases}
$$

with the DLVO charge given by $Z_{>}=Z \exp (\bar{\kappa} a) /(1+\bar{\kappa} a)$, and with the parameter $A$ defined by

$$
A=4 \pi \beta w_{0} \frac{n}{\bar{\kappa}^{3}}\left[(1+\bar{\kappa} a)^{2} e^{-2 \bar{\kappa} a}+(\bar{\kappa} a)^{2}-1\right] .
$$

The effective pair interaction $V(r)$ is very similar to the traditional DLVO potential $V_{2}(r)$ of Eq. (1), but with two important differences. The first difference involves the screening parameter $\bar{\kappa}$ in $V(r)$, which is to be contrasted with the reservoir screening parameter $\kappa$ in $V_{2}(r)$. The second difference is that the amplitude of $V(r)$ is enhanced compared to $V_{2}(r)$ by a factor $(1+A)$. This can be traced back to our particular choice of linear combinations of density profiles that we used to solve the Euler-Lagrange equations.

In Fig. 2, we plot $A$ as a function of the screening parameter $\bar{\kappa} a$ for several values of the packing fraction $\eta$. The plot shows that $A \ll 1$ for essentially all packing fractions of interest here. Moreover, one can also show that $A \equiv 0$ if the hard potentials are defined as $U(\mathbf{r})=V(\mathbf{r})+2 \bar{\rho}_{\overline{+}} W(\mathbf{r}) /\left(\bar{\rho}_{+}\right.$ $\left.+\bar{\rho}_{-}\right)$instead of the definition used here. This latter choice does not affect any of the other volume terms, but does involve another choice for $v_{0}$ and $w_{0}$, and does change the expression of $\bar{\phi}$ (see Appendix B). For these two reasons we set $A \equiv 0$ in the remainder of the paper.

The so-called volume terms $\Phi_{\mathrm{D}}$ and $\Phi_{0}$ are very similar to their canonical counterparts that were derived in Ref. [47]. The main difference is that the present volume term includes the term $-\int \mathrm{d} \mathbf{r}\left[\mu_{+} \rho_{+}(\mathbf{r})+\mu_{-} \rho_{-}(\mathbf{r})\right]$ due to the grand-canonical character of our calculations. This leads to another difference, since one should now view the Hamiltonian $H$ as a function of $n$ and the reservoir salt concentration $c_{\mathrm{s}}$, i.e., one 
should take the dependence of $\Phi$ on $\bar{\rho}_{ \pm}$and $\bar{\kappa}$ as a dependence on $c_{\mathrm{s}}$ and $n$ through Eqs. (34) and (25). It is the nontrivial (and nonlinear) dependence of $\beta \Phi_{\mathrm{D}} / V$ and $\beta \Phi_{0} / V$ on the colloid density $n$, at fixed $c_{\mathrm{s}}$, which is responsible for interesting thermodynamic effects, as we will see later.

\section{THERMODYNAMICS}

\section{A. Free energy}

As we have now found the functional form (36) for the effective Hamiltonian of the colloids, we are ready to calculate the corresponding free energy $F\left(N, V, T, c_{\mathrm{s}}\right)$ defined just below Eq. (13). From this, the other thermodynamic quantities follow. Since the volume terms in (36) are independent of the coordinates of the colloids, we can factor out their Boltzmann weights and write

$$
\exp (-\beta F)=\exp [-\beta \Phi] \operatorname{tr}_{\mathrm{c}} \exp \left[-\beta \sum_{i<j}^{N} V\left(R_{i j}\right)\right] .
$$

This can be rewritten as

$$
F=\Phi_{\mathrm{D}}+\Phi_{0}+F_{\text {id }}+F_{\text {exc }},
$$

with $\Phi_{\mathrm{D}}$ and $\Phi_{0}$ defined in Eq. (38), with the colloidal idealgas free energy

$$
F_{\text {id }}=N k_{\mathrm{B}} T[\ln (n \mathcal{V})-1],
$$

and where $F_{\text {exc }}$ is the nonideal (excess) free energy due to the colloid-colloid pair interactions (39). Here we calculate $F_{\text {exc }}$ variationally, using the Gibbs-Bogoliubov inequality [74-77]. This inequality states that the excess free energy $F_{\text {exc }}^{(\text {ref }}$ of a so-called reference system of volume $V$ that contains $N$ particles with any pair interaction $V^{(\text {ref })}\left(R_{i j}\right)$, satisfies

$$
F_{\text {exc }} \leqslant F_{\text {exc }}^{\text {(ref) }}+\left\langle\sum_{i<j}\left[V\left(R_{i j}\right)-V^{(\mathrm{ref})}\left(R_{i j}\right)\right]\right\rangle_{\text {ref }},
$$

where $\langle\cdots\rangle_{\text {ref }}$ denotes a thermodynamic average that is to be evaluated in the reference system. The key idea is to use a reference pair potential with a free parameter with respect to which the right-hand side of Eq. (44) can be minimized; the minimum is then the optimal estimate for the free energy $F_{\text {exc }}$ of interest. We use two different reference system to calculate the free energy of fluid and crystal phases, respectively.

For the fluid phase we use a hard-sphere reference system, with the hard-sphere diameter $d$ as variational parameter. Introducing the effective hard-sphere packing fraction $\xi$ $=(\pi / 6) n d^{3}$, we can write

$$
\frac{F_{\mathrm{exc}}}{N k_{\mathrm{B}} T}=\min _{d}\left\{\frac{4 \xi-3 \xi^{2}}{(1-\xi)^{2}}+\frac{n}{2} 4 \pi \int_{d}^{\infty} \mathrm{d} r r^{2} g_{d}(r ; \xi) \beta V(r)\right\},
$$

where the first term is Carnahan-Starling expression for the hard-sphere free energy [74,78], and where $g_{d}(r ; \xi)$ is the radial distribution function of a fluid of hard spheres with diameter $d$ and packing fraction $\xi$. We approximate $g_{d}(r ; \xi)$ by the Verlet-Weis corrected Percus-Yevick expressions
$[74,79]$. This allows for an analytic evaluation of the integral in Eq. (45), since the Yukawa form of $V(r)$ turns this integral into a Laplace transform of $r g_{d}(r ; \xi)$, for which accurate expressions have been derived on the basis of Padé fits in Refs. $[80,81]$. The minimization with respect to $d$ is then numerically performed straightforwardly. Note that such a minimum indeed exists, as the excess free energy of the hard-sphere reference system becomes infinitely large in the limit of large particle sizes. Because the particles in our actual system have also a hard core of radius $a$, we impose that $d \geqslant 2 a$.

As a reference system for the solid phase, we use $N$ classical Einstein oscillators [82,83] on an fcc lattice [84]. The Einstein frequency $\omega_{\mathrm{E}}$ plays the role of the variational parameter used to minimize the right-hand side of Eq. (44). For the thermodynamic average of the Yukawa interactions in this system, we use the expressions found by Shih et al. [84].

Thus far, we have only considered fcc configurations for the solid phase, but there is no principal problem to generalize this to other structures, such as bcc [47]. Consequently, we only consider gas-liquid, fluid-fec and fcc-fcc phase equilibriums in this paper.

\section{B. Osmotic pressure}

The osmotic pressure $\Pi=P-2 c_{\mathrm{s}} k T$ of the suspension under consideration follows from $P=-\partial F / \partial V$ at fixed $N$ and $c_{\mathrm{s}}$. We can therefore use our expression for $F$ given in Eq. (42) to obtain $\Pi\left(n, c_{\mathrm{s}}\right)$ explicitly as

$$
\Pi=\Pi_{\mathrm{D}}+\Pi_{0}+\Pi_{\mathrm{id}}+\Pi_{\mathrm{exc}}
$$

with $\Pi_{\mathrm{D}}=-2 c_{\mathrm{s}} k_{\mathrm{B}} T-\left(\partial \Phi_{\mathrm{D}} / \partial V\right)$, the Van 't Hoff (ideal-gas) contribution $\Pi_{\mathrm{id}}=-\left(\partial F_{\mathrm{id}} / \partial V\right)=n k_{\mathrm{B}} T$, the excess pressure $\Pi_{\text {exc }}=-\left(\partial F_{\text {exc }} / \partial V\right)$, and the remaining term $\Pi_{0}=-\left(\partial \Phi_{0} / \partial V\right)$. Explicit general expressions for $\Pi_{D}, \Pi_{0}$, and $\Pi_{\text {exc }}$ can, of course, be given on the basis of Eqs. (38) and (37), and, e.g., (45), respectively, but it turns out to be instructive to focus on these expressions in the limit of point colloids with radius $a=0$ (such that $\eta=0$ ): this reduces the algebra and allows for an interesting illustration of cancellations of some of the electrostatic contributions to the osmotic pressure $\Pi$. We stress, however, that we used the full expressions in our numerical calculations presented below.

In the point-colloid limit, we have

$$
\begin{aligned}
\beta \Pi_{\mathrm{D}} & =-2 c_{\mathrm{s}}+\bar{\rho}_{+}+\bar{\rho}_{-}=-2 c_{\mathrm{s}}+\sqrt{(Z n)^{2}+\left(2 c_{\mathrm{s}}\right)^{2}} \\
& = \begin{cases}\frac{(Z n)^{2}}{4 c_{\mathrm{s}}}+\mathcal{O}\left(n^{4}\right) & Z n \ll 2 c_{\mathrm{s}} \\
Z n-2 c_{\mathrm{s}}+\mathcal{O}\left(c_{\mathrm{s}}^{2}\right) & Z n \gg 2 c_{\mathrm{s}},\end{cases}
\end{aligned}
$$

and a little tedious but straightforward algebra yields

$$
\beta \Pi_{0}= \begin{cases}-\frac{(Z n)^{2}}{4 c_{\mathrm{s}}}+\mathcal{O}\left(n^{3}\right) & Z n \ll 2 c_{\mathrm{s}} \\ -b n^{3 / 2}+\mathcal{O}\left(c_{\mathrm{s}}^{2}\right) & Z n \gg 2 c_{\mathrm{s}},\end{cases}
$$

with a coefficient $b=\sqrt{Z \pi \lambda_{B}} Z^{2} \lambda_{B} / 2$. We focus first on the low-density/high-salt regime $Z n \ll 2 c_{\text {s }}$ and then on the opposite regime. 
The expressions (47) and (48) show a cancellation of the dominant term in the regime $Z n \ll 2 c_{s}$, such that in this regime $\Pi \simeq \Pi_{\mathrm{id}}+\Pi_{\mathrm{exc}}$, i.e., the pressure is actually the pressure of the effective one-component system described by the pairwise screened-Coulomb Hamiltonian. Interestingly, however, one can also write the virial expansion $\beta F_{\text {exc }} / V=B_{2}(\bar{\kappa}) n^{2}$ $+\mathcal{O}\left(n^{3}\right)$ in this regime, where the second virial coefficient $[74]$ is

$$
B_{2}(\bar{\kappa})=\frac{1}{2} \int \mathrm{d} \mathbf{r}(1-\exp [-\beta V(r)]),
$$

with the colloidal pair potential $V(r)$ defined in Eq. (39). In the limit of weak interactions, the exponent in Eq. (49) can be linearized with the result that $B_{2}=Z^{2} / 4 c_{\mathrm{s}}$ for point colloids. This means that $\beta \Pi_{\mathrm{exc}} \simeq(Z n)^{2} / 4 c_{\mathrm{s}} \simeq \beta \Pi_{\mathrm{D}}$ and, hence, that the pressure can also be approximated by the Donnan expression $\Pi \simeq \Pi_{\mathrm{id}}+\Pi_{\mathrm{D}}$. In other words, on the basis of this simple analysis one expects "reliable" results for the pressure (and, hence, for the thermodynamics) in the regime $Z n$ $\ll 2 c_{\mathrm{s}}$ by taking either the full four-term expression (46) for $\Pi$, or the two-term expressions $\Pi_{\mathrm{id}}+\Pi_{\mathrm{D}}$ and $\Pi_{\mathrm{id}}+\Pi_{\mathrm{exc}}$, but not any other combination. This will be confirmed by our numerical results below.

The situation is bit more complicated in the opposite lowsalt regime $2 c_{\mathrm{s}} \ll Z n$, since then (i) no cancellations take place and (ii) the virial expansion for $F_{\text {exc }}$ breaks down because of the long-range character of the unscreenedCoulomb interactions.

As a simple approximation for highly charged particles (specifically, particles for which $Z^{2} \lambda_{\mathrm{B}} / a \gg 1$ ), the pair correlation function $g_{d}(r)$ can be set to $g_{d}(r)=1$ for $r \gtrsim 1 / 2 n^{-1 / 3}$ and to 0 otherwise. One can then show that the lowest-order contribution to the excess pressure takes the form $\beta \Pi_{\mathrm{exc}}$ $=-b^{\prime} n^{4 / 3}$ with $b^{\prime}=\pi Z^{2} \lambda_{\mathrm{B}} / 12$. As a consequence, we find the asymptotic low-salt result

$$
\beta \Pi=(1+Z) n-b^{\prime} n^{4 / 3}-b n^{3 / 2},
$$

which contains Donnan, colloidal-pair, and Debye-Hückellike contributions. The prefactors of the fractional powers would change if a proper Güntelberg charging process would have been performed [85], but the present analysis is good enough to capture the spinodal instability that is now well known to be realistic for primitive model systems at sufficiently strong coupling (low enough temperature) [86-92]. On this basis one could expect that the present theory predicts phase separation in low-salt colloidal suspensions. Within the full theory for $F$ we, indeed, find this phenomenon in Sec. V.

We now illustrate our results for the osmotic pressure by numerically comparing the theoretically predicted values to experimental measurements in Fig. 3. The experimental system is an ethanol suspension of colloidal silica spheres, for which $\Pi(n)$ was determined by integration of the measured density profile in sedimentation equilibrium [72,73]. The system parameters are $Z=32,2 c_{\mathrm{s}}=16 \mu \mathrm{M}, \lambda_{\mathrm{B}}=2.38 \mathrm{~nm}$, and $a=21.9 \mathrm{~nm}$. Since $Z \lambda_{\mathrm{B}} / a \approx 3$, we do not expect too much charge renormalization, and as $Z n / 2 c_{\mathrm{s}} \approx 0.7$ at the highest density considered here $(\eta=0.01)$, this experiment is ex-

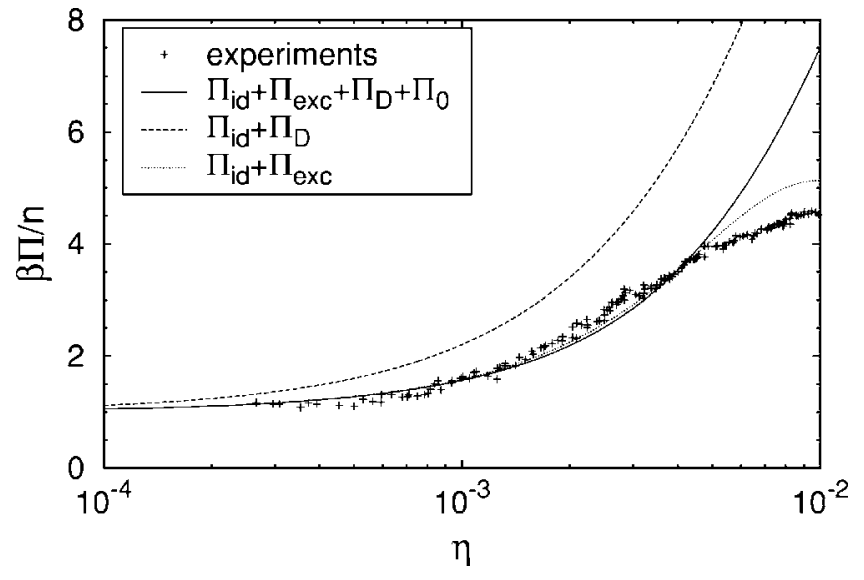

FIG. 3. Equation of state compared to the experiments of Raşa et al. [72], described by the parameters $Z=32$ for the colloidal charge, $a / \lambda_{\mathrm{B}}=96.7$ for the radius-to-Bjerrum length ratio, and $2 c_{\mathrm{S}}$ $=16 \mu \mathrm{M}$ for the reservoir salt concentration. Shown are the experimental data (crosses), a one-component DLVO system (dotted line), the Donnan theory (dashed line), and our full linear theory (solid line). Two of the three theoretical curves describe the experimental curves accurately for $\eta \lessgtr 0.003$, the Donnan theory is less accurate although still qualitatively reasonable in this regime.

pected to be in the high-salt regime where not only the full expression (46) for $\Pi$ but also both the one-component expressions $\Pi \simeq \Pi_{\mathrm{id}}+\Pi_{\mathrm{exc}}$ and the Donnan expression $\Pi$ $\simeq \Pi_{\mathrm{id}}+\Pi_{\mathrm{D}}$ are expected to "work" with reasonable accuracy.

This is to some extent confirmed by Fig. 3, where the measured osmotic pressure is in quantitative agreement with two of the three theoretical versions at low packing fractions $\eta \lesssim 0.003$ or so; the Donnan pressure is less accurate. At higher densities the different theoretical curves deviate from each other (and from the experiment), with the onecomponent result $\Pi_{\mathrm{id}}+\Pi_{\mathrm{exc}}$ being closest to the actual experiment. A word of caution is appropriate here, however, since recent work by Biesheuvel indicates that charge regularization is relevant in the present system, i.e., the bare colloidal charge $Z$ is not a constant but decreases with density, where significant deviations of the low-density charge is predicted for $\eta \geq 0.002$ [93]. This is rather precisely the regime where the theories begin to deviate from the experiment. This issue will also be addressed in more detail in future work.

From the fact that the one-component osmotic pressure $\Pi=\Pi_{\mathrm{id}}+\Pi_{\mathrm{exc}}$ describes the experimental data rather accurately, one may conclude that the experimentally found "inflated" profiles of Ref. [73] need not necessarily be described by theories, such as those of Ref. [94], where a threecomponent mixture (cations, anions, and colloids) in gravity gives rise to an ion-entropy-induced self-consistent electric field that lifts the colloids to higher altitudes than expected on the basis of their mass. The equation of state suggests that an alternative description could be given, based on hydrostatic equilibrium of an effective one-component system of colloidal spheres with pairwise screened-Coulomb repulsions only. The latter picture is not in contradiction with the existence of the electric field, since the density variation with height implies a variation of the Donnan potential with 


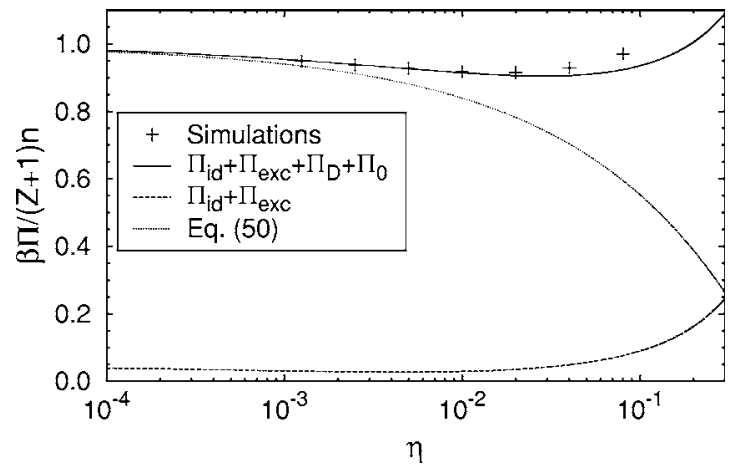

FIG. 4. Equation of state (compressibility factor) compared to the computer simulation of Ref. [95], where $Z=40$ and $a / \lambda_{\mathrm{B}}$ $=22.5$. Shown are the simulation data from [95] (crosses), the pressure $\Pi$ from our full linear theory (solid line), the approximate low-salt expression (50) (dotted line), and the pairwise onecomponent result $\Pi_{\mathrm{id}}+\Pi_{\text {exc }}$ (dashed line). The theoretical curves are based on the reservoir salt concentration $c_{\mathrm{s}}=10^{-15} \mathrm{M}$, which is low enough to ensure an essentially vanishing coion concentration in all state points shown here.

height through Eq. (24). The two pictures are, in this sense, merely two sides of the same coin, at least on length scales beyond which the local density approximation applies that underlies the one-component theory. On smaller length scales the source of this electric field involves deviations from local charge neutrality, which cannot be explained by hydrostatic equilibrium and a bulk equation of state alone.

The other system for which we calculate the osmotic pressure is one of the systems that Linse studied by Monte Carlo simulations in Ref. [95]. This system is free of added salt, contains colloids with a charge $Z=40$ and a radius-toBjerrum length parameter of $a / \lambda_{\mathrm{B}}=22.5$ for monovalent ions (in the notation of Ref. [95] the coupling parameter is $\Gamma_{I I}$ $=0.0445)$. The simulated results are shown in Fig. 4, together with several versions of the present theory. It is clear that the major contribution to the osmotic compressibility factor originates from the pressure $\Pi_{\mathrm{id}}+\Pi_{\mathrm{D}} \approx(Z+1) n$, which exceeds the one-component combination $\Pi_{\mathrm{id}}+\Pi_{\text {exc }}$ by at least an order of magnitude. The decrease of $\beta \Pi / n$ for $\eta \lesssim 0.02$ is due to the contribution $\Pi_{0}$. Our calculated pressure describes the simulation data quite well, showing that volume terms may have a pronounced effect on the thermodynamic properties of low-salt suspensions, while the pairwise DLVO picture without volume terms breaks down qualitatively. We note, finally, that the limiting expression (50) for the pressure in the limit for point colloids can be seen to catch the lowdensity negative curvature of $\beta \Pi / n$ with $n$ as predicted by the full theory and the simulations, but not the increased stability at higher $n$.

\section{PHASE DIAGRAMS}

From the free energy per unit volume $f(n)=F / V$ at fixed $c_{\mathrm{s}}$, we calculate the chemical potential and the pressure, and we impose the usual condition of thermodynamic equilibrium (11) to find a phase equilibrium. We already mentioned that this is numerically much less involved than in the ca-

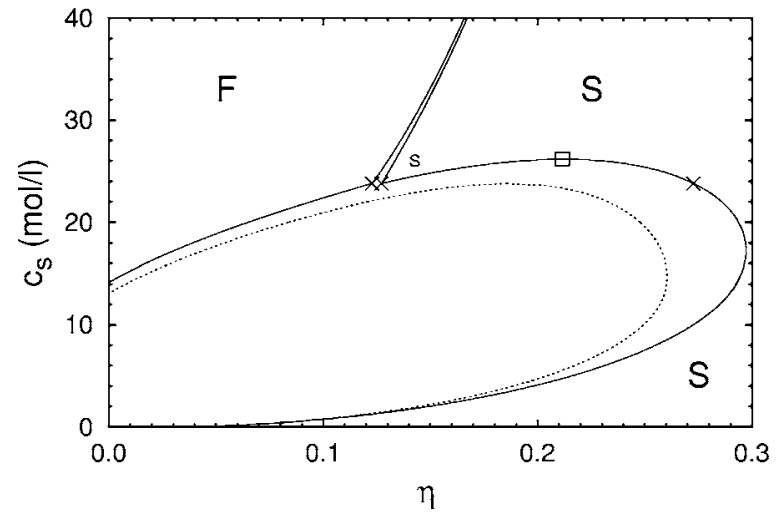

FIG. 5. Phase diagram for a colloidal suspension as a function of colloidal packing fraction $\eta$ and reservoir salt concentration $c_{\mathrm{s}}$. The colloidal radius and charge are $a=326 \mathrm{~nm}$ and $Z=7300$, and the solvent is water at room temperature such that the Bjerrum length is $\lambda_{\mathrm{B}}=0.72 \mathrm{~nm}$. The solid lines denote fluid-solid and solidsolid binodals, and the dotted line shows the underlying metastable gas-liquid binodal. The fluid-solid-solid triple point is denoted by $\times$, and the solid-solid critical point by $\square$.

nonical calculations of, e.g., Ref. [47], where the set (8) is to be solved. We merely illustrate the feasibility of these calculations here by showing two phase diagrams, for a particular $Z, a$, and $\lambda_{\mathrm{B}}$. In forthcoming publications we will fully exploit the relative simplicity of the grand-canonical formulation of the theory by "scanning" the full parameter space $\left(Z, \lambda_{\mathrm{B}} / a\right)$, including a generalization of the present theory to include charge renormalization [96].

The first set of parameters that we consider is $Z=7300$, $a=326 \mathrm{~nm}$, and $\lambda_{\mathrm{B}}=0.72 \mathrm{~nm}$, which corresponds to the experiments of Ref. [25]. The phase diagram that follows from the present theory is displayed in Fig. 5, and shows phase coexistence with a considerable or large density gap at $c_{\mathrm{S}}$ $\lesssim 20 \mu \mathrm{M}$, and only a very small density gap at higher $c_{\mathrm{s}}$. At a salt concentrations of about $23.8 \mu \mathrm{M}$, a liquid-solid-solid triple point occurs (denoted by $\times$ in Fig. 5), and at $26.2 \mu \mathrm{M}$ a solid-solid critical point is located (denoted by $\square$ in the figure). Although somewhat difficult to see in this picture, there is no lower critical point.

The phase diagram of Fig. 5 is pretty similar to the one calculated in Ref. [47] using the canonical version of the theory [47], but with a few substantial differences. The canonical theory, for instance, does not find any solid-solid coexistence, nor does it find a triple point for these parameters. Also the canonical theory predicts a lower critical point, while the current grand-canonical version of the theory does not. Despite this differences the main phenomenon is shared that, at low salinity $c_{\mathrm{s}} \lesssim 20 \mu \mathrm{M}$, a density gap opens up.

The physical mechanism for this demixing transitions into a dilute and dense phase is identical to what was explained in Refs. $[47,97,98]$ : the self energy of the double layers as represented by the third term of the volume term $\Phi_{0}$ in Eq. (38) drives a spinodal instability at low enough $c_{\mathrm{s}}$, even though the pair interactions are purely repulsive. The underlying physical mechanism is that the cohesive energy that stabilizes the dense phase stems from the compression of the 


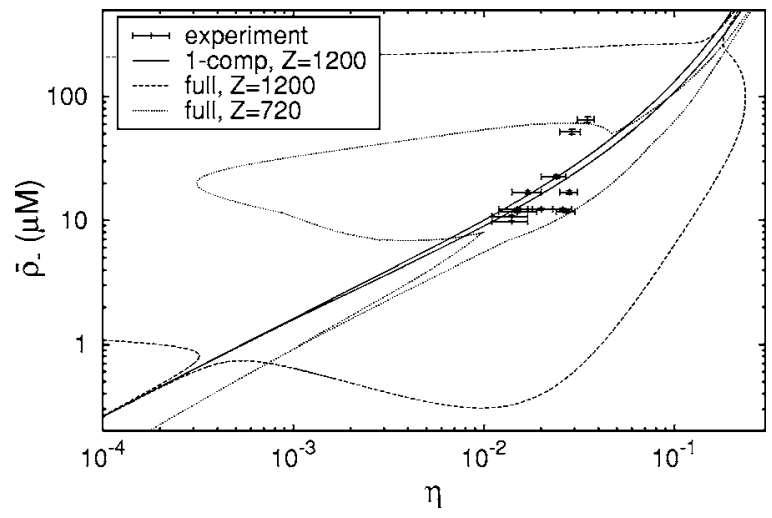

FIG. 6. Phase diagrams for the parameters of the experiment by Monovoukas and Gast [100]. The Bjerrum length for this system is $\lambda_{\mathrm{B}}=0.72 \mathrm{~nm}$, and the partical radius is $a=66.7 \mathrm{~nm}$. The data points plotted here correspond to the samples for which a fluid-solid coexistence was observed in Ref. [100]. The solid line is a phase diagram for a one-component DLVO system, the dashed line denotes the phase boundaries for our full linear theory for a charge of $Z=1200$. The dotted line gives the phase boundary for the same full linear theory, but with a lower charge $Z=720$.

double layer's thickness $\bar{\kappa}^{-1}$ upon increasing the colloid density: this effect brings the charge in the diffuse double layer closer to the oppositely charged colloidal surface. This mechanism is very similar to the one that causes gas-liquid demixing in the restrictive primitive model according to Debye-Hückel theory $[87,99]$.

A word of caution is appropriate here: given that $Z \lambda_{\mathrm{B}} / a$ $\simeq 16$, one expects a substantial renormalization of the charge within nonlinear Poisson-Boltzmann theory for this system and, hence, a reduction of the tendency to demix. Whether or not this mechanism for phase separation remains strong enough to yield a big density gap in the phase diagram if charge renormalization is taken into account, will be investigated in a future publication [59].

The second phase diagram that we present here is for the parameters of the experiments of Monovoukas and Gast [100], where $Z=1200, a=66.7 \mathrm{~nm}$, and $\lambda_{\mathrm{B}}=0.72 \mathrm{~nm}$. These parameters were chosen because the experiments reveal a significant density gap, by a factor of three, between the coexisting fluid and solid phases at salinity of the order of $10 \mu \mathrm{M}$. Such a large density gap cannot be explained by the DLVO pair potential alone, and hence, we investigate here to what extent the volume terms may account for this effect.

The phase diagram, shown in the $\eta-\bar{\rho}_{-}$representation in Fig. 6, shows the experimental points and three fluid-solid binodals based on the present theory. As the results of Ref. [100] seem to be independent of the concentration of added salt for salt concentration lower than $\sim 8 \mu \mathrm{M}$, we assumed an extra background salt concentration of $8 \mu \mathrm{M}$ for the experimental points. Note that this representation of the phase diagram, with the vertical axis representing the concentration of added salt instead of the reservoir concentration, is such that the tie lines (which have been omitted for clarity) are no longer horizontal as in the $\eta-c_{\mathrm{S}}$ representation, but instead tilted to lower $\bar{\rho}_{-}$at higher $\eta$ due to the Donnan effect (see also Fig. 1).
The first binodal in Fig. 6 is the one based on the ideal and excess part of $F$ only, i.e., we assume that $\Phi_{0}$ and $\Phi_{\mathrm{D}}$ vanish (or more accurately: the volume terms are assumed to be merely linear in $N$ and $V$ and do therefore not affect the phase diagram). Although this binodal gives a fair representation of the experimental points (probably this is how $Z$ $=1200$ was chosen), they do not capture the large density gap. The second binodal is based on the full expression for $F$, including the volume terms, with $Z=1200$. We find an enormous density gap that is much larger than experimentally observed, and that extends to unreasonably high salt concentrations. The third binodal is also based on our expression for $F$ with volume terms, but now for a smaller charge $Z=720$. Interestingly, this choice gives a density-gap at fluidsolid coexistence in the right salt concentration regime, but the magnitude of the gap is yet much bigger than experimentally observed. The reduction of the charge from $Z=1200$ to $Z=720$ may give a rough idea of the effect of charge renormalization and shows that this nonlinear effect reduces the tendency to demix considerably. Theories for charge renormalization $[50,53]$ show that the renormalized charge is actually not a constant but depends on the screening parameter and the density; the present value $Z=720$ corresponds to the dilute limit value at $\kappa a \approx 0.8$, i.e., at $c_{\mathrm{s}} \approx 10 \mu \mathrm{M}$, and is fixed here for simplicity. We expect this to be a reasonable lower limit for the renormalized charge in the region in which the phase separation occurs. Also this system will be investigated within a nonlinear version of Poisson-Boltzmann theory in a future publication [59].

\section{CONCLUSION}

We have reformulated and rederived the volume term theory for suspensions of charged colloids [46,47]. Our present derivation should be more transparent than the original one, for instance because we can now avoid the extra parameter $\lambda$ that regulates the Coulomb potential from $1 / r$ to $\exp (-\lambda r) / r$ with $\lambda \rightarrow 0$ only at the end of the calculation. Moreover, the presently derived expressions should be easier to use in numerical calculations of thermodynamic properties and phase diagrams because the ions are treated grandcanonically instead of canonically, thereby assuring equal chemical potential of the ions from the outset. Moreover, a direct connection with Donnan theory is now made, with explicit expressions for the Donnan potential and the ion concentration in the system. In future publications we will fully exploit the computational advantages and extend the theory to include charge renormalization.

We derived analytic expressions for the osmotic pressure in the point-colloid limit for both the low-salinity and highsalinity limits. The low-salinity limit of the pressure was shown to correspond to the Donnan expression, while in the limit of high salt concentrations the traditional DLVO results are recovered. The present full theory interpolates between these results and gives a good account of measured and simulated osmotic pressures in both regimes.

We also calculated two phase diagrams. The first one matches the parameters of Ref. [47] and shows a similarly large phase instability at low salinity, although there are also 
a few substantial differences. The second phase diagram matches the parameters of the experiments by Monovoukas and Gast [100], where an anomalously large density gap at fluid-solid coexistence was reported. Interestingly, the present theory does predicts a density gap at fluid-solid coexistence, but its magnitude is much larger than experimentally observed. We stress, however, that these phase diagrams are calculated in a regime where charge renormalization cannot be ignored. The relative transparency of the present derivation allows to systematically include this nonlinear effect into the theory, as will be shown in a future publication [59].

The linear theory described in this paper already shows, however, that volume terms can affect the osmotic pressure of low-salt suspensions qualitatively, also in regimes where charge renormalization and other nonlinear effects are not expected to be important.

\section{ACKNOWLEDGMENTS}

It is a pleasure to thank Mircea Raşa and Albert Philipse for collaborations and sharing their osmotic pressure data with us. This work is part of the research program of the "Stichting voor Fundamenteel Onderzoek der Materie (FOM)," which is financially supported by the "Nederlandse Organisatie voor Wetenschappelijk Onderzoek (NWO)."

\section{APPENDIX A: THE GRAND POTENTIAL}

In this appendix, we derive the equilibrium grand potential $\Omega$. We show that upon insertion of this grand potential into Eq. (13), the effective Hamiltonian can be cast into the form specified by Eqs. (36)-(39).

In the framework of density functional theory, the equilibrium grand potential is given by the minimum of the functional $\Omega\left[\rho_{+}, \rho_{-}\right]$of Eq. (20). This minimum is found by inserting the Euler-Lagrange equation (21) into the functional. This leads to the following expression for the grand potential:

$$
\begin{aligned}
\frac{\beta \Omega}{V}= & \sum_{ \pm} \bar{\rho}_{ \pm}\left(\ln \left[\frac{\bar{\rho}_{ \pm}}{c_{\mathrm{s}}}\right]-1\right)+\frac{Z n}{2} \bar{\phi}+\eta \beta w_{0} \frac{\bar{\rho}_{+}+\bar{\rho}_{-}}{2} \\
& +\frac{1}{2 V} \int \mathrm{d} \mathbf{r} \rho(\mathbf{r}) \beta V(\mathbf{r})+\frac{1}{2 V} \int \mathrm{d} \mathbf{r} \beta W(\mathbf{r})\left[\rho_{+}(\mathbf{r})+\bar{\rho}_{-}(\mathbf{r})\right] .
\end{aligned}
$$

The "electrostatic" integral can be evaluated as

$$
\begin{aligned}
\frac{1}{2 V} \int \mathrm{d} \mathbf{r} \rho(\mathbf{r}) \beta V(\mathbf{r})= & \frac{1}{2 V} \frac{1}{(2 \pi)^{3}} \int \mathrm{d} \mathbf{k} \beta V_{\mathbf{k}} \rho_{-\mathbf{k}} \\
= & -\frac{1}{2} \frac{1}{1-\eta} \frac{\left(\bar{\rho}_{+}-\bar{\rho}_{-}\right)^{2}}{\bar{\rho}_{+}+\bar{\rho}_{-}}-\frac{Z n}{2} \bar{\phi}-\frac{n}{2} \frac{Z^{2} \bar{\kappa} \lambda_{\mathrm{B}}}{1+\bar{\kappa} a} \\
& +\frac{1}{V} \sum_{i<j}\left\{\left(1+\frac{A}{2}\right)\left(\frac{Z e^{\bar{\kappa} a}}{1+\bar{\kappa} a}\right)^{2} \frac{\lambda_{\mathrm{B}} e^{-\bar{\kappa} R_{i j}}}{R_{i j}}\right\} \\
& -\frac{1}{V} \sum_{i<j}\left\{-Z^{2} \frac{\lambda_{\mathrm{B}}}{R_{i j}}\right\},
\end{aligned}
$$

where we inserted the Fourier transform $\rho_{\mathbf{k}}$ of $\rho(\mathbf{r})$ from (33), and the Fourier transform $V_{\mathbf{k}}$ of $V(\mathbf{r})$, which is given by $\beta V_{\mathbf{k}}=-\frac{4 \pi}{k^{3}}\left\{\left(\beta v_{0}+Z \frac{\lambda_{\mathrm{B}}}{a}\right) k a \cos k a-\beta v_{0} \sin k a\right\} \sum_{j} e^{i \mathbf{k} \cdot \mathbf{R}_{j}}$.

The factor $A / 2$ in the fourth term on the right-hand side of Eq. (A2) is caused by the expulsion of microionic charges from the colloid cores, and is given by Eq. (40). Note that the first and second terms of Eq. (A2) result from the $\propto k^{2} \delta(\mathbf{k})$ term in Eq. (33), which are did not contribute to the charge density.

In a similar way, the hard-core part of the grand potential (A1) is evaluated as

$$
\begin{aligned}
& \frac{1}{2 V} \int \mathrm{d} \mathbf{r} \beta W(\mathbf{r})\left[\rho_{+}(\mathbf{r})+\rho_{-}(\mathbf{r})\right] \\
& =\frac{1}{2 V} \frac{1}{(2 \pi)^{3}} \frac{\bar{\rho}_{+}-\bar{\rho}_{-}}{\bar{\rho}_{+}+\bar{\rho}_{-}} \int \mathrm{d} \mathbf{k} \beta W_{\mathbf{k}} \rho_{-\mathbf{k}} \\
& =\frac{1}{V} \frac{A}{2}\left(\frac{Z e^{\bar{\kappa} a}}{1+\bar{\kappa} a}\right)^{2} \sum_{i<j} \frac{\lambda_{\mathrm{B}} e^{-\bar{\kappa} R_{i j}}}{R_{i j}},
\end{aligned}
$$

where the Fourier transform of $W(\mathbf{r})$ is given by Eq. (30) and where we used that $W(\mathbf{r})\left[\rho_{+}(\mathbf{r}) / \bar{\rho}_{+}+\rho_{-}(\mathbf{r}) / \bar{\rho}_{-}\right] \equiv 0$.

Substitution of Eqs. (A2) and (A4) into the grand potential (A1) leads to

$$
\begin{aligned}
\beta \Omega= & (1+A)\left(\frac{Z e^{\bar{\kappa} a}}{1+\bar{\kappa} a}\right)^{2} \sum_{i<j} \lambda_{\mathrm{B}} \frac{e^{-\bar{\kappa} R_{i j}}}{R_{i j}} \\
& -Z^{2} \sum_{i<j} \frac{\lambda_{\mathrm{B}}}{R_{i j}}+\beta \Phi
\end{aligned}
$$

where the "volume term" $\Phi=\Phi_{\mathrm{D}}+\Phi_{0}$ is given by Eqs. (37) and (38).

Gathering Eqs. (13), (6), and (A5), we find that the effective Hamiltonian can be cast into the form given by Eqs. (36)-(39).

\section{APPENDIX B: ALTERNATIVE HARD-CORE TERMS}

We have already mentioned that, in this paper, we use a slightly different definition of the hard-core parameters $\beta v_{0}$ and $\beta w_{0}$ than were used by van Roij and Hansen in [46] and van Roij et al. [47]. In this appendix, we make this statement explicit and calculate, within the grand-canonical scheme of this work, the effective Hamiltonian using the definition of the hard-core potentials of Refs. [46,47].

In contrast to the presently used definition $U_{ \pm}(\mathbf{r})= \pm V(\mathbf{r})$ $+W(\mathbf{r})$ for the microion-colloid interactions, as outlined just above Eq. (19a), van Roij and Hansen used the definition

$$
U_{ \pm}(\mathbf{r})= \pm V(\mathbf{r})+\frac{2 \bar{\rho}_{\overline{+}}}{\bar{\rho}_{+}+\bar{\rho}_{-}} W(\mathbf{r}),
$$

where the potentials $V(\mathbf{r})$ and $W(\mathbf{r})$ are defined in Eqs. (19a) and $(19 b)$. With this definition, the grand potential becomes 


$$
\begin{aligned}
\Omega\left[\rho_{+}, \rho_{-}\right]= & \Omega_{\mathrm{id}}^{\prime}\left[\rho_{+}\right]+\Omega_{\mathrm{id}}^{\prime}\left[\rho_{-}\right]+\int \mathrm{d} \mathbf{r} \rho(\mathbf{r}) V(\mathbf{r}) \\
& +k_{\mathrm{B}} T \lambda_{\mathrm{B}} \int \mathrm{d} \mathbf{r} \mathrm{d} \mathbf{r}^{\prime} \frac{\rho(\mathbf{r}) \rho\left(\mathbf{r}^{\prime}\right)}{\left|\mathbf{r}-\mathbf{r}^{\prime}\right|} \\
& +\frac{2 \bar{\rho}_{+} \bar{\rho}_{-}}{\bar{\rho}_{+}+\bar{\rho}_{-}} \int \mathrm{d} \mathbf{r}\left(\frac{\rho_{+}(\mathbf{r})}{\bar{\rho}_{+}}+\frac{\rho_{-}(\mathbf{r})}{\bar{\rho}_{-}}\right) W(\mathbf{r}),
\end{aligned}
$$

where the ideal-gas functionals $\beta \Omega_{\mathrm{id}}\left[\rho_{ \pm}\right]$are defined in Eq. (18).

The corresponding Euler-Lagrange equations are then given by

$$
\ln \frac{\bar{\rho}_{ \pm}}{c_{\mathrm{S}}}+\frac{\rho_{ \pm}(\mathbf{r})-\bar{\rho}_{ \pm}}{\bar{\rho}_{ \pm}} \pm \phi(\mathbf{r})+\frac{2 \bar{\rho}_{\overline{+}} \beta W(\mathbf{r})}{\bar{\rho}_{+}+\bar{\rho}_{-}}=0 .
$$

By integrating these equations over the system volume, and using the condition for global charge neutrality, we find that the average densities $\bar{\rho}_{ \pm}$are identical to those given in Eq. (25). The Donnan potential $\bar{\phi}$, however, is not given by Eq. (24) anymore, but by

$$
\bar{\phi}=-\sinh ^{-1}\left[\frac{Z n}{2 c_{\mathrm{s}}} e^{\eta \beta w_{0}}\right]+\eta \beta w_{0} \frac{Z n}{\bar{\rho}_{+}+\bar{\rho}_{-}}
$$

instead. Although this expression also reduces to the usual Donnan expression in the limit $n \rightarrow 0$, it is physically less satisfactory than the result we found in Eq. (24), as at high $\eta$ its sign can become different from that of the colloidal charge $-Z e$.

To calculate the density profiles, we take the following linear combination of the Euler-Lagrange equations (B3):

$$
\begin{gathered}
\frac{\rho_{+}(\mathbf{r})}{\bar{\rho}_{+}}+\frac{\rho_{-}(\mathbf{r})}{\bar{\rho}_{-}}=2\left[1-\beta W(\mathbf{r})+\eta \beta w_{0}\right] ; \\
\frac{\rho(\mathbf{r})-\bar{\rho}}{\bar{\rho}_{+}+\bar{\rho}_{-}}=-[\phi(\mathbf{r})-\bar{\phi}] .
\end{gathered}
$$

Note that, due to the different definition of $U_{ \pm}(\mathbf{r})$, the hardcore potential $W(\mathbf{r})$ is now totally decoupled from the charge density $\rho(\mathbf{r})$.

Equation (B5a) is identical to Eq. (26a); thus, its solution is again 0 inside the hard cores of the colloids and given by Eq. (28) outside the hard cores, provided that we fix $\beta w_{0}$ $=1 /(1-\eta)$.

The second equation (B5b) is not identical to its counterpart, Eq. (26b). The solution is quite similar though: we need to fix the hard-core parameter $\beta v_{0}$ to

$$
\beta v_{0}=-Z \frac{\bar{\kappa} \lambda_{\mathrm{B}}}{1+\bar{\kappa} a}
$$

in order to make sure that the charge density $\rho(\mathbf{r})$ is a multicentered sum of DLVO profiles. The solution (in k-space) is then given by

$$
\begin{aligned}
\rho_{\mathbf{k}}= & (2 \pi)^{3}\left\{\bar{\rho}+\left(\bar{\rho}_{+}+\bar{\rho}_{-}\right) \bar{\phi}\right\} \frac{k^{2}}{k^{2}+\bar{\kappa}^{2}} \delta(\mathbf{k}) \\
& +\frac{Z}{1+\bar{\kappa} a} \frac{\cos k a+\frac{\bar{\kappa}}{k} \sin k a}{1+k^{2} / \bar{\kappa}^{2}} \sum_{j} e^{i \mathbf{k} \cdot \mathbf{R}_{j},}
\end{aligned}
$$

which, in real space, is indeed a multicentered sum $\rho(\mathbf{r})$ $=\sum_{i} \rho_{1}\left(\left|\mathbf{r}-\mathbf{R}_{i}\right|\right)$ with the individual profiles given by Eq. (35). Note that Eqs. (33) and (B7) only differ in the $\propto k^{2} \delta(\mathbf{k})$ term. As a consequence, the profiles $\rho(\mathbf{r})$ resulting from those two equations, are identical, but the minimum of the functional differs.

Upon insertion of the equilibrium density profiles into the functional (B2), we immediately note that the last term on the right-hand side vanishes. The grand potential then becomes

$$
\begin{aligned}
\frac{\beta \Omega}{V}= & \sum_{ \pm} \bar{\rho}_{ \pm}\left(\ln \left[\frac{\bar{\rho}_{ \pm}}{c_{\mathrm{s}}}\right]-1\right)+\frac{Z n}{2} \bar{\phi}+\frac{\eta}{1-\eta} \frac{2 \bar{\rho}_{+} \bar{\rho}_{-}}{\bar{\rho}_{+}+\bar{\rho}_{-}} \\
& +\frac{1}{2 V} \int \operatorname{dr} \rho(\mathbf{r}) \beta V(\mathbf{r}) .
\end{aligned}
$$

The integral in this expression can now be calculated using Parseval's theorem and the expression (A3) for the Fourier transform of $V(\mathbf{r})$. The result is

$$
\begin{aligned}
\frac{1}{2 V} \int \mathrm{d} \mathbf{r} \rho(\mathbf{r}) \beta V(\mathbf{r})= & \frac{1}{V} \sum_{i<j}\left\{\left(\frac{Z e^{\bar{\kappa} a}}{1+\bar{\kappa} a}\right)^{2} \frac{\lambda_{\mathrm{B}} e^{-\bar{\kappa} R_{i j}}}{R_{i j}}-Z^{2} \frac{\lambda_{\mathrm{B}}}{R_{i j}}\right\} \\
& -\frac{n}{2} \frac{Z^{2} \bar{\kappa} \lambda_{\mathrm{B}}}{1+\bar{\kappa} a}-\frac{1}{2} \frac{\left(\bar{\rho}_{+}-\bar{\rho}_{-}\right)^{2}}{\bar{\rho}_{+}+\bar{\rho}_{-}}-\frac{Z n}{2} \bar{\phi},
\end{aligned}
$$

so that the grand potential eventually becomes

$$
\beta \Omega=\left(\frac{Z e^{\bar{\kappa} a}}{1+\bar{\kappa} a}\right)^{2} \sum_{i<j} \lambda_{\mathrm{B}} \frac{e^{-\bar{\kappa} R_{i j}}}{R_{i j}}-Z^{2} \sum_{i<j} \frac{\lambda_{\mathrm{B}}}{R_{i j}}+\beta \Phi .
$$

The volume term $\beta \Phi$ is exactly equal to the one that was found previously in Eqs. (37) and (38); the colloidal pair interaction, however, reduces to a purely DLVO interaction, i.e., the factor $A$ we found before, is now equal to 0 .
[1] S. Asakura and F. Osawa, J. Chem. Phys. 22, 1255 (1954).

[2] A. Vrij, Pure Appl. Chem. 48, 471 (1976).

[3] Y. Levin, Rep. Prog. Phys. 65, 1577 (2002).

[4] J.-P. Hansen and H. Löwen, Annu. Rev. Phys. Chem. 51, 209
(2000).

[5] C. N. Likos, Phys. Rep. 348, 267 (2001).

[6] M. Dijkstra and R. van Roij, Phys. Rev. Lett. 89, 208303 (2002). 
[7] J. M. Brader, R. Evans, M. Schmidt, and H. Löwen, J. Phys.: Condens. Matter 14, L1 (2001).

[8] C. Russ, H. H. von Grünberg, M. Dijkstra, and R. van Roij, Phys. Rev. E 66, 011402 (2002).

[9] B. Derjaguin and L. Landau, Acta Physicochim. URSS 14, 633 (1941).

[10] J. W. Verwey and J. T. G. Overbeek, Theory of the Stability of Lyotropic Colloids (Elsevier, Amsterdam, 1948).

[11] B. J. Alder and T. E. Wainwright, J. Chem. Phys. 27, 1208 (1957).

[12] P. N. Pusey, W. van Megen, P. Bartlett, B. J. Ackerson, J. G. Rarity, and S. M. Underwood, Phys. Rev. Lett. 63, 2753 (1989).

[13] M. J. Stevens and M. O. Robbins, Europhys. Lett. 12, 81 (1990).

[14] A.-P. Hynninen and M. Dijkstra, Phys. Rev. Lett. 94, 138303 (2005).

[15] V. Reus, L. Belloni, T. Zemb, N. Lutterbach, and H. Vesmold, J. Phys. II 7, 603 (1997).

[16] V. Reus, L. Belloni, T. Zemb, N. Lutterbach, and H. Versmold, Colloids Surf., A 151, 449 (1999).

[17] E. B. Sirota, H. D. Ou-Yang, S. K. Sinha, P. M. Chaikin, J. D. Axe, and Y. Fujii, Phys. Rev. Lett. 62, 1524 (1989).

[18] S. H. Chen, E. Y. Sheu, J. Kalus, and H. Hoffman, J. Appl. Crystallogr. 21, 751 (1988).

[19] C. P. Royall, M. E. Leunissen, and A. van Blaaderen, J. Phys.: Condens. Matter 15, S3581 (2003).

[20] J. C. Crocker and D. G. Grier, Phys. Rev. Lett. 73, 352 (1994).

[21] K. Vondermassen, J. Bongers, A. Mueller, and H. Versmold, Langmuir 10, 1351 (1994).

[22] K. Ito, H. Hiroshi, and N. Ise, Science 263, 66 (1994).

[23] B. V. R. Tata, E. Yamahara, P. V. Rajamani, and N. Ise, Phys. Rev. Lett. 78, 2660 (1997).

[24] N. Ise, T. Okubo, M. Sugimura, K. Ito, and H. Nolte, J. Chem. Phys. 78, 536 (1983).

[25] A. E. Larsen and D. G. Grier, Nature (London) 385, 230 (1997).

[26] B. V. R. Tata, M. Rajalakshmi, and A. K. Arora, Phys. Rev. Lett. 69, 3778 (1992).

[27] T. Palberg and M. Würth, Phys. Rev. Lett. 72, 786 (1994).

[28] B. V. R. Tata and A. K. Arora, Phys. Rev. Lett. 72, 787 (1994).

[29] A.-P. Hynninen, M. Dijkstra, and R. v. Roij, J. Phys.: Condens. Matter 15, S3549 (2003).

[30] A.-P. Hynninen, M. Dijkstra, and R. van Roij, Phys. Rev. E 69, 061407 (2004).

[31] C. Russ, M. Brunner, C. Bechinger, and H. H. von Grünberg, Europhys. Lett. 69, 468 (2005).

[32] C. Russ, K. Zahn, and H.-H. von Grünberg, J. Phys.: Condens. Matter 15, S3509 (2003).

[33] K. Zahn, G. Maret, C. Russ, and H. H. von Grünberg, Phys. Rev. Lett. 91, 115502 (2003).

[34] M. Brunner, J. Dobnikar, H. H. von Grunberg, and C. Bechinger, Phys. Rev. Lett. 92, 078301 (2004).

[35] J. Dobnikar, M. Brunner, H. H. von Grunberg, and C. Bechinger, Phys. Rev. E 69, 031402 (2004).

[36] A.-P. Hynninen and M. Dijkstra J. Chem. Phys. 123, 244902 (2005).

[37] M. Dijkstra and R. van Roij, J. Phys.: Condens. Matter 10, 1219 (1998).

[38] P. B. Warren, J. Chem. Phys. 112, 4683 (2000).
[39] A. Diehl, M. C. Barbosa, and Y. Levin, Europhys. Lett. 53, 86 (2001).

[40] P. B. Warren, e-print cond-mat/0006289.

[41] A. R. Denton, Phys. Rev. E 62, 3855 (2000).

[42] S. N. Petris and D. Y. C. Chan, J. Chem. Phys. 116, 8588 (2002).

[43] D. Y. C. Chan, P. Linse, and S. N. Petris, Langmuir 17, 4202 (2001).

[44] P. B. Warren, J. Phys.: Condens. Matter 15, S3467 (2003).

[45] B. Beresford-Smith, D. Y. C. Chan, and D. J. Mitchell, J. Colloid Interface Sci. 105, 216 (1985).

[46] R. van Roij and J.-P. Hansen, Phys. Rev. Lett. 79, 3082 (1997).

[47] R. van Roij, M. Dijkstra, and J.-P. Hansen, Phys. Rev. E 59, 2010 (1999).

[48] F. G. Donnan, Chem. Rev. (Washington, D.C.) 1, 73 (1924).

[49] J. T. G. Overbeek, Prog. Biophys. Biophys. Chem. 6, 57 (1956).

[50] S. Alexander, P. M. Chaikin, P. Grant, G. Morales, P. Pincus, and D. Hone, J. Chem. Phys. 80, 5776 (1984).

[51] E. Trizac and Y. Levin, Phys. Rev. E 69, 031403 (2004).

[52] Y. Levin, E. Trizac, and L. Bocquet, J. Phys.: Condens. Matter 15, S3523 (2003).

[53] E. Trizac, L. Bocquet, M. Aubouy, and H. von Grünberg, Langmuir 19, 4027 (2003).

[54] L. Belloni, Colloids Surf., A 140, 227 (1998).

[55] L. Bocquet, E. Trizac, and M. Aubouy, J. Chem. Phys. 117, 8138 (2002).

[56] Y. Levin, M. C. Barbosa, and M. N. Tamashiro, Europhys. Lett. 41, 123 (1998).

[57] M. N. Tamashiro and H. Schiessel, J. Chem. Phys. 119, 1855 (2003).

[58] H. H. von Grünberg, R. van Roij, and G. Klein, Europhys. Lett. 55, 580 (2001).

[59] B. Zoetekouw and R. van Roij (unpublished).

[60] R. Evans, Adv. Phys. 28, 143 (1979).

[61] R. Evans, Fundamentals of Inhomogeneous Fluids (Marcel Dekker, New York, 1992), pp. 85-175.

[62] H. Löwen, J. Phys.: Condens. Matter 14, 11897 (2002).

[63] R. A. Marcus, J. Chem. Phys. 23, 1057 (1955).

[64] M. Fushiki, J. Chem. Phys. 97, 6700 (1992).

[65] H. Löwen, P. A. Madden, and J. P. Hansen, Phys. Rev. Lett. 68, 1081 (1992).

[66] H. Löwen, J.-P. Hansen, and P. A. Madden, J. Chem. Phys. 92, 3275 (1993).

[67] J. Dobnikar, Y. Chen, R. Rzehak, and H. H. von Grünberg, J. Phys.: Condens. Matter 15, S263 (2003).

[68] J. Dobnikar, Y. Chen, R. Rzehak, and H. H. von Grünberg, J. Chem. Phys. 119, 4971 (2003).

[69] J. Dobnikar, R. Rzehak, and H. H. von Grünberg, Europhys. Lett. 61, 695 (2003).

[70] M. Deserno and H.-H. von Grünberg, Phys. Rev. E 66, 011401 (2002).

[71] M. Deserno and C. Holm, Electrostatic Effects in Soft Matter and Biophysics, NATO Science Series II: Mathematics, Physics and Chemistry Vol. 46 (Kluwer, Dordrecht, 2002).

[72] M. Raşa, B. H. Erné, B. Zoetekouw, R. van Roij, and A. P. Philipse, J. Phys.: Condens. Matter 17, 2293 (2005).

[73] M. Raşa and A. P. Philipse, Nature (London) 429, 857 (2004).

[74] J.-P. Hansen and I. R. McDonald, Theory of Simple Liquids 
(Academic Press, London, 1986).

[75] A. Isihara, J. Phys. A 1, 539 (1968).

[76] J. W. Gibbs, Elementary Principles in Statistical Mechanics (Oxford University Press, London, 1902), Chap. XI.

[77] N. N. Bogoliubov, Dokl. Akad. Nauk SSSR 119, 244 (1954).

[78] N. F. Carnahan and K. E. Starling, J. Chem. Phys. 51, 635 (1969).

[79] L. Verlet and J.-J. Weis, Phys. Rev. A 5, 939 (1972).

[80] S. B. Yuste and A. Santos, Phys. Rev. A 43, 5418 (1991).

[81] S. Bravo Yuste, M. López de Haro, and A. Santos, Phys. Rev. E 53, 4820 (1996).

[82] J. M. Ziman, Theory of Solids, 2nd ed. (Cambridge University Press, Cambridge, England, 1972).

[83] N. W. Ashcroft and N. D. Mermin, Solid State Physics (Saunders, Philadelphia, 1976).

[84] W. Y. Shih, I. A. Aksay, and R. Kikuchi, J. Chem. Phys. 86, 5127 (1987).

[85] Y. Levin, J. Chem. Phys. 113, 9722 (2000).

[86] A.-P. Hynninen, M. Dijkstra, and A. Z. Panagiotopoulos, J. Chem. Phys. 123, 84903 (2005).
[87] M. E. Fisher and Y. Levin, Phys. Rev. Lett. 71, 3826 (1993). [88] J. M. Caillol, D. Levesque, and J. J. Weis, J. Chem. Phys. 107, 1565 (1997).

[89] G. Orkoulas and A. Z. Panagiotopoulos, J. Chem. Phys. 110, 1581 (1999).

[90] Q. Yan and J. J. de Pablo, J. Chem. Phys. 111, 9509 (1999).

[91] Y. Levin and M. E. Fisher, Physica A 225, 164 (1995).

[92] A. Z. Panagiotopoulos and M. E. Fisher, Phys. Rev. Lett. 88, 045701 (2002).

[93] P. M. Biesheuvel, J. Phys.: Condens. Matter 16, L499 (2004).

[94] R. van Roij, J. Phys.: Condens. Matter 15, S3569 (2003).

[95] P. Linse, J. Chem. Phys. 113, 4359 (2000).

[96] B. Zoetekouw and R. van Roij (unpublished).

[97] R. van Roij and R. Evans, J. Phys.: Condens. Matter 11, 10047 (1999).

[98] R. van Roij, J. Phys.: Condens. Matter 12, A263 (2000).

[99] M. E. Fisher, J. Phys.: Condens. Matter 8, 9103 (1996).

[100] Y. Monovoukas and A. P. Gast, J. Colloid Interface Sci. 128, 533 (1989). 